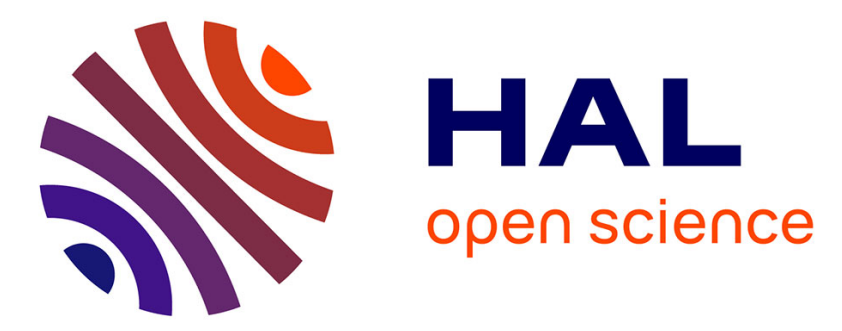

\title{
Stability analysis of split-explicit free surface ocean models: implication of the depth-independent barotropic mode approximation
}

Jeremie Demange, Laurent Debreu, Patrick Marchesiello, Florian Lemarié, Eric Blayo, Christopher Eldred

\section{To cite this version:}

Jeremie Demange, Laurent Debreu, Patrick Marchesiello, Florian Lemarié, Eric Blayo, et al.. Stability analysis of split-explicit free surface ocean models: implication of the depth-independent barotropic mode approximation. Journal of Computational Physics, 2019, 398 (108875), pp.1-26. 10.1016/j.jcp.2019.108875 . hal-01947706v2

\section{HAL Id: hal-01947706 \\ https://hal.inria.fr/hal-01947706v2}

Submitted on 17 Dec 2019

HAL is a multi-disciplinary open access archive for the deposit and dissemination of scientific research documents, whether they are published or not. The documents may come from teaching and research institutions in France or abroad, or from public or private research centers.
L'archive ouverte pluridisciplinaire HAL, est destinée au dépôt et à la diffusion de documents scientifiques de niveau recherche, publiés ou non, émanant des établissements d'enseignement et de recherche français ou étrangers, des laboratoires publics ou privés. 


\title{
Stability analysis of split-explicit free surface ocean models: implication of the depth-independent barotropic mode approximation.
}

\author{
Jérémie Demange $^{\mathrm{a}}$, Laurent Debreu ${ }^{\mathrm{a}, *}$, Patrick Marchesiello ${ }^{\mathrm{b}}$, Florian Lemariéa ${ }^{\text {, Eric Blayo }}{ }^{\mathrm{a}}$, Christopher \\ Eldred $^{\mathrm{a}}$ \\ ${ }^{a}$ Univ. Grenoble Alpes, Inria, CNRS, Grenoble INP*, LJK, 38000 Grenoble, France \\ * Institute of Engineering Univ. Grenoble Alpes \\ ${ }^{b}$ IRD/LEGOS, Toulouse, France
}

\begin{abstract}
The evolution of the oceanic free-surface is responsible for the propagation of fast surface gravity waves, which approximatively propagate at speed $\sqrt{g H}$ (with $g$ the gravity and $H$ the local water depth). In the deep ocean, this phase speed is roughly two orders of magnitude faster than the fastest internal gravity waves. The very strong stability constraint imposed by those fast surface waves on the time-step of numerical models is handled using a mode splitting between slow (internal / baroclinic) and fast (external / barotropic) motions to allow the possibility to adopt specific numerical treatments in each component. The barotropic mode is traditionally approximated by the vertically integrated flow because it has only slight vertical variations. However the implications of this assumption on the stability of the splitting are not well documented. In this paper, we describe a stability analysis of the mode splitting technique based on an eigenvector decomposition using the true (depth-dependent) barotropic mode. This allows us to quantify the amount of dissipation required to stabilize the approximative splitting. We show that, to achieve stable integrations, the dissipation usually applied through averaging filters can be drastically reduced when incorporated at the level of the barotropic time stepping. The benefits are illustrated by numerical experiments. In addition, the formulation of a new mode splitting algorithm using the depth-dependent barotropic mode is introduced.
\end{abstract}

Keywords: Ocean models, Barotropic mode, Mode splitting, Stability analysis

2010 MSC: 00-01, 99-00

\section{Introduction}

Almost all current ocean circulation models have now relaxed the rigid lid approximation and integrate a prognostic equation for the free surface evolution. In this case, external gravity waves which roughly propagate at speed $\sqrt{g H}$ (with $g$ the gravity and $H$ the water depth) are explicitly simulated. Because a tridimensional implicit time stepping algorithm seems computationally impractical, explicit time stepping schemes are preferred; however, the numerical integration of the fastest external waves then introduces a strong stability constraint on the model time step. Under several assumptions (including a flat bottom), the linear stratified primitive equations can be projected onto a set of orthogonal normal modes. This projection highlights a fast (external or barotropic) mode and slow (internal or baroclinic) modes. The usual approach

\footnotetext{
*Corresponding author. E-mail address: Laurent.Debreu@inria.fr (L. Debreu)
} 
for time integration in state-of-the-art oceanic models is to introduce a mode splitting, where the barotropic mode is integrated separately from the baroclinic modes. In order to satisfy the stability condition, the barotropic part can be integrated using either a 2D implicit time stepping algorithm [1] or a time splitting approach [2] where the 2D barotropic mode is evolved with small sub-time steps. Here we only consider the time splitting approach, which is less prone to dispersion errors. It seems likely that the approach presented here could also be applied to the case of implicit time stepping for the barotropic mode, but this is not pursued. A usual assumption [2] consists in assuming that the external mode is vertically constant (i.e. depth independent). However, although depth-averaging provides an accurate approximation to the barotropic mode, this leads to a non orthogonal separation of slow and fast modes, even in the linear case. A reconciliation of the estimates of the barotropic mode coming from the $2 \mathrm{D}$ barotropic integration and the $3 \mathrm{D}$ baroclinic integration is thus needed, and stability of the splitting scheme separating fast and slow modes is not guaranteed. In addition to this inexact splitting, several other reasons motivate the need for some form of time filtering $[3,4]$. They are described below.

To be more specific, let us introduce the nonlinear barotropic 2D $x-z$ system:

$$
\left\{\begin{aligned}
\frac{\partial \bar{u}}{\partial t}+g \frac{\partial \eta}{\partial x} & =-\frac{1}{\rho_{0}} \frac{\partial}{\partial x} \frac{1}{H+\eta} \int_{-H}^{\eta} p_{h} \mathrm{~d} z+G(u) \\
\frac{\partial \eta}{\partial t}+\frac{\partial(H+\eta) \bar{u}}{\partial x} & =0
\end{aligned}\right.
$$

where $\bar{u}$ is the depth averaged velocity, $\eta$ the elevation of the free surface, and the right hand side is the sum of the depth integrated internal pressure gradient and $G(u)$ the vertical average of all other terms (i.e. nonlinear advection, diffusion ...). $\rho_{0}$ is the constant reference density and $g$ the gravity. This right hand side of (1) is held constant during the barotropic integration (i.e. the $N_{\text {split }}$ sub-time steps corresponding to one time step for the baroclinic part). However the free surface $\eta$ evolves during these barotropic sub-time steps, which makes the coherence of the vertical integral problematic, since its upper bound is $\eta$. An efficient remedy based on a redefinition of the barotropic pressure-gradient terms to account for the local variations in density field is proposed in [3]. Moreover, aliasing errors due to nonlinear advection are an additional source of instability, which is controlled by time averaging. The beneficial effect of recomputing the fast barotropic part of this term at each sub-time steps has been studied in [5].

To prevent instabilities associated to these splitting errors, a time filtering of the barotropic variables has to be applied. This can be achieved by using a diffusive time stepping algorithm in the barotropic integration itself [2]. But in practice, most ocean models perform this time-filtering using explicit averaging filters (see $[6],[4]$ and $\S 4.1)$ involving the sub-time step instantaneous barotropic solutions, before reconciliation with the $3 \mathrm{D}$ parts.

Among all the splitting errors described above, it is not yet well understood which source of error is the most damaging for stability (and consequently which error justifies the amount of extra diffusion introduced through time-filtering). One objective of this paper deals with the impact of the aforementioned inexact splitting on the stability of numerical models. In $[7,8,9]$, detailed numerical analysis of various $2 \mathrm{D} / 3 \mathrm{D}$ time stepping algorithms are presented in two-layers models. Those studies specifically looked at the impact of the 2D/3D time stepping algorithms and of adding diffusion in the 2D part of a numerical model. However, 
the impact of the depth-independent barotropic mode assumption is not explicitly highlighted and this is precisely the objective of this work. Another objective is to provide a general framework for the stability analysis of the mode splitting approach. Unlike previous studies, our analysis is based on a projection of the solution on the basis formed by non-approximate (depth-dependent) barotropic and baroclinic modes. This framework should allow the design of efficient 2D/3D time stepping algorithms.

The paper is organized as follows. We first briefly recall the classical theory of vertical mode decomposition and introduce important parameters for the stability analysis. Then we proceed to the stability analysis, focusing on the amount of diffusion required to control the instability arising from the depth-independent barotropic mode assumption. We then compare the choice of averaging filters and diffusion within the 2D time integration scheme. Idealized numerical experiments are performed and confirm the large amount of diffusion introduced by the averaging filters. More realistic experiments (nonlinear equations, non flat bottom) of internal tides generation are then presented with similar conclusions.

\section{Normal mode decomposition}

\subsection{Projection onto normal modes}

First, we briefly recall the normal mode decomposition theory (e.g $[10,11])$. We consider a linearization of the $2 \mathrm{D}(\mathrm{x}-\mathrm{z})$ primitive equations (i.e. under the Boussinesq and hydrostatic assumptions) around a motionless state in hydrostatic equilibrium and in absence of rotation:

$$
\begin{aligned}
\frac{\partial u}{\partial t}+\frac{1}{\rho_{0}} \frac{\partial p}{\partial x} & =0 \\
\frac{\partial p}{\partial z} & =-g \rho \\
\frac{\partial u}{\partial x}+\frac{\partial w}{\partial z} & =0 \\
\frac{\partial \rho}{\partial t}+w \frac{\mathrm{d} \bar{\rho}}{\mathrm{d} z} & =0
\end{aligned}
$$

In the vertical direction the model extends from the flat bottom $z=-H$ to the top given by the free surface elevation $z=\eta(x, t)$. Here $u(x, z, t)$ and $w(x, z, t)$ denote the perturbation components of horizontal and vertical fluid velocities (primes are omitted for clarity). $p(x, z, t)$ and $\rho(x, z, t)$ denote pressure and density perturbations around a state $(\bar{p}(z), \bar{\rho}(z))$ satisfying the hydrostatic relation $\frac{\mathrm{d} \bar{p}(z)}{\mathrm{d} z}=-\bar{\rho}(z) g$ where $\bar{\rho}(z)$ is a reference density profile and $\rho_{0}$ its depth-averaged value. This density profile is assumed to be stable, i.e. $\frac{\mathrm{d} \bar{\rho}}{\mathrm{d} z}(z)<0$.

The linearized $(\eta \ll H)$ surface and bottom boundary conditions read

$$
\begin{aligned}
\frac{\partial \eta}{\partial t} & =w(z=0) & \text { at } z=0 \\
w & =0 & \text { at } z=-H \\
p(z=0) & =\rho_{0} g \eta & \text { at } z=0
\end{aligned}
$$


The solution of (2)-(5) can be decomposed using vertical modes $M_{q}(z)([11])$

$$
\begin{aligned}
& u(x, z, t)=\sum_{q=0}^{\infty} u_{q}(x, t) M_{q}(z) \\
& p(x, z, t)=\rho_{0} g \sum_{q=0}^{\infty} h_{q}(x, t) M_{q}(z)
\end{aligned}
$$

Inserting these decompositions respectively in (3), (4),(7), and (8) implies:

$$
\begin{aligned}
\rho(x, z, t) & =-\rho_{0} \sum_{q=0}^{\infty} h_{q}(x, t) \frac{\mathrm{d} M_{q}}{\mathrm{~d} z}(z) \\
w(x, z, t) & =-\sum_{q=0}^{\infty} \frac{\partial u_{q}}{\partial x}(x, t) \int_{-H}^{z} M_{q}(s) \mathrm{d} s \\
\eta(x, t) & =\sum_{q=0}^{\infty} h_{q}(x, t) M_{q}(0)
\end{aligned}
$$

(4) can be rewritten as

$$
\frac{\partial u}{\partial x}-\frac{1}{\rho_{0}} \frac{\partial}{\partial z}\left(N^{-2}(z) \frac{\partial}{\partial z}\right)\left(\frac{\partial p}{\partial t}\right)(z, t)=0
$$

with $N(z)=\left(-\frac{g}{\rho_{0}} \frac{\mathrm{d} \bar{\rho}}{\mathrm{d} z}\right)^{1 / 2}$ the Brünt-Vaisala frequency (which is positive, since the reference density profile is stable). This equation, along with appropriate boundary conditions coming from (6) and (7), demonstrates that $M_{q}(z)$ are the eigenvectors of the following Sturm Liouville problem:

$$
\begin{aligned}
\Lambda M_{q}(z) & =\lambda_{q} M_{q}(z) \quad-H<z<0 \\
\frac{\mathrm{d} M_{q}}{\mathrm{~d} z}(-H) & =0 \\
\frac{\mathrm{d} M_{q}}{\mathrm{~d} z}(0) & =-\frac{N^{2}(0)}{g} M_{q}(0)
\end{aligned}
$$

where $\Lambda=-\frac{\mathrm{d}}{\mathrm{d} z}\left(N^{-2}(z) \frac{\mathrm{d}}{\mathrm{d} z}\right)$. Since $\Lambda$ is a compact symmetric operator, it admits a basis of orthonormal eigenvectors $M_{q}(z)$ with positive eigenvalues $\lambda_{q}\left(0 \leq \lambda_{0}<\lambda_{1}<\ldots\right)$. The vertical modes are orthonormal with respect to the scalar product $\langle f, g\rangle=\frac{1}{H} \int_{-H}^{0} f(z) g(z) \mathrm{d} z$ (the vertical integration extends from $-H$ to 400 instead of $\eta$ in this linear framework), so that we can write $u_{q}=\left\langle u, M_{q}\right\rangle$, and $h_{q}=\frac{1}{\rho_{0} g}\left\langle p, M_{q}\right\rangle$. Since the $\lambda_{q}$ s have the same units as $\Lambda$, i.e. $m^{-2} \cdot s^{2}$ (see (15)), we will denote $\lambda_{q}=1 / c_{q}^{2}$ in the following. $c_{q}$ is the phase speed associated to the $q^{\text {th }}$ mode (in particular, $c_{0}$ is the speed of external gravity waves).

The time evolution of $u_{q}$ and $h_{q}$ are then obtained as follows:

- The scalar product of $(2)$ and $M_{q}(z)$ (i.e. multiplying $(2)$ by $M_{q}(z)$ and integrating vertically over $[-H, 0])$ leads to

$$
\frac{\partial u_{q}}{\partial t}+g \frac{\partial h_{q}}{\partial x}=0
$$

- The scalar product of (14) and $M_{q}(z)$ leads to

$$
\frac{\partial h_{q}}{\partial t}+\frac{c_{q}^{2}}{g} \frac{\partial u_{q}}{\partial x}=0
$$


We thus get a set of uncoupled systems for $q=0,1 \ldots$ :

$$
\begin{aligned}
\frac{\partial u_{q}}{\partial t}+g \frac{\partial h_{q}}{\partial x} & =0 \\
\frac{\partial h_{q}}{\partial t}+\frac{c_{q}^{2}}{g} \frac{\partial u_{q}}{\partial x} & =0
\end{aligned}
$$

We will now investigate further the structure of the modes $M_{q}$, starting with the simplified case of a constant stratification.

\subsection{Vertical modes in the case of a constant background stratification}

In this paragraph, we consider the particular case of a constant background stratification $\mathrm{d} \bar{\rho} / \mathrm{d} z(\mathrm{i} . e . \rho$ is an affine function of $z$ ). Let us introduce the dimensionless parameter $\epsilon=N^{2} H / g$ whose typical value is such that $\epsilon \ll 1$ (due to the Boussinesq hypothesis). Indeed, for $N=10^{-3} \mathrm{~s}^{-1}$ and $H=4000 \mathrm{~m}$, we get $\epsilon \approx 4.10^{-4}$. The normal mode definition (15-17) can be expressed in terms of $\epsilon$ (considering $\left.\lambda_{q}=c_{q}^{-2}\right)$

$$
\left\{\begin{array}{l}
-\frac{\mathrm{d}^{2} M_{q}}{\mathrm{~d} z^{2}}=\epsilon \frac{g}{c_{q}^{2} H} M_{q} \\
\frac{\mathrm{d} M_{q}}{\mathrm{~d} z}(-H)=0 \\
\frac{\mathrm{d} \bar{M}_{q}}{\mathrm{~d} z}(0)=-\frac{\epsilon}{H} M_{q}(0)
\end{array}\right.
$$

The analytical solution of this Sturm-Liouville problem is:

$$
\begin{array}{r}
M_{q}(z)=M_{q}(0)\left[\cos \left(\sqrt{\epsilon} \frac{\sqrt{g H}}{c_{q}} \frac{z}{H}\right)-\sqrt{\epsilon} \frac{c_{q}}{\sqrt{g H}} \sin \left(\sqrt{\epsilon} \frac{\sqrt{g H}}{c_{q}} \frac{z}{H}\right)\right] \\
\text { with } \tan \left(\sqrt{\epsilon} \frac{\sqrt{g H}}{c_{q}}\right)=\sqrt{\epsilon} \frac{c_{q}}{\sqrt{g H}}
\end{array}
$$

The values $c_{0}, c_{1}, \ldots$ can be found by solving (22). It is easy to see that the positive roots $r_{q}$ of the equation $\tan Y=\epsilon / Y$ are located in each interval $(q \pi ; q \pi+\pi / 2)$. Hence $c_{q}=\sqrt{\epsilon} \frac{\sqrt{g H}}{r_{q}}$.

The fastest mode (i.e. $q=0$ ) is called the barotropic mode. Since $\epsilon \ll 1, r_{0}$ is close to 0 , which means that $c_{0}$ is large. A Taylor expansion of (22) w.r.t. $\epsilon$ leads to

$$
c_{0}=\sqrt{g H}\left(1+\frac{\epsilon}{6}\right)+\mathcal{O}\left(\epsilon^{2}\right)
$$

A Taylor expansion of (21) then yields

$$
M_{0}(z)=M_{0}(0)\left(1-\epsilon\left[\frac{z}{H}+\frac{z^{2}}{2 H^{2}}\right]+\mathcal{O}\left(\epsilon^{2}\right)\right)
$$

Using the fact that $\left\langle M_{0}, M_{0}\right\rangle=1$, one gets a Taylor expansion of $M_{0}(0)$, which finally leads to

$$
\left\{\begin{array}{l}
c_{0}=\alpha_{0} \sqrt{g H} \text { with } \alpha_{0}=1+\frac{\epsilon}{6}+\mathcal{O}\left(\epsilon^{2}\right) \\
M_{0}(z)=1-\epsilon\left[\frac{1}{3}+\frac{z}{H}+\frac{z^{2}}{2 H^{2}}\right]+\mathcal{O}\left(\epsilon^{2}\right)
\end{array}\right.
$$

It is then clear that the barotropic mode is not equal to the constant function 1, corresponding to the velocity $\sqrt{g H}$, but slightly departs from it. The other values of $c_{q}(q \geq 1)$ are obtained in the same way. A Taylor expansion of (22) yields $r_{q}=q \pi+\frac{\epsilon}{q \pi}+\mathcal{O}\left(\epsilon^{2}\right)$. We can then find the expression of the so-called baroclinic modes:

$$
q \geq 1, \quad\left\{\begin{array}{l}
c_{q}=\alpha_{q} \sqrt{g H} \quad \text { with } \alpha_{q}=\frac{\sqrt{\epsilon}}{q \pi}+\mathcal{O}\left(\epsilon^{3 / 2}\right) \\
M_{q}(z)=\sqrt{2}\left[\cos \left(\frac{q \pi}{H} z\right)-\frac{\epsilon}{q \pi} \sin \left(\frac{q \pi}{H} z\right)\right]+\mathcal{O}\left(\epsilon^{2}\right)
\end{array}\right.
$$




\subsection{Non constant background stratification case}

The stability analysis performed in the next sections will essentially use the fact that $\alpha_{0}$ is close to, and greater than, 1. However the knowledge of its exact value, obtained in the previous paragraph in the case of constant $N$, is not required. In the case of non constant $N$, a good approximation of $c_{0}^{2} /(g H)$ has been given in [12] (eq. (29)). Under the hydrostatic and Boussinesq assumptions, applying the same technique as in [12] leads (at first order in $\epsilon$ ) to

$$
\alpha_{0}=\frac{c_{0}}{\sqrt{g H}}=\sqrt{1+\frac{1}{g H^{2}} \int_{-H}^{0}\left(\int_{z}^{0} N^{2}\left(z^{\prime}\right)\left(H+z^{\prime}\right) \mathrm{d} z^{\prime}\right) \mathrm{d} z}
$$

which proves that $\alpha_{0}>1$. Note that, in the case of constant $N$, this expression $(25)$ gives $\alpha_{0}=\sqrt{1+\frac{1}{3} \frac{N^{2} H}{g}}=$ $\sqrt{1+\frac{\epsilon}{3}}$, which matches our development (23).

In the following we will also use the equalities (which hold for non constant $N$ ):

$$
\begin{aligned}
\frac{1}{H} \int_{-H}^{0} M_{q}(z) \mathrm{d} z & =\frac{M_{q}(0)}{\lambda_{q} g H}=\alpha_{q}^{2} M_{q}(0) \\
\sum_{q=0}^{+\infty}\left[\frac{1}{H} \int_{-H}^{0} M_{q}(z) \mathrm{d} z\right]^{2} & =1
\end{aligned}
$$

The first equality (26) is obtained by vertically integrating the normal mode definition (15). The second equality (27) is obtained by writing the decomposition of a constant velocity field $1=u=\sum_{q=0}^{+\infty} u_{q} M_{q}$, which leads to $u_{q}=<1, M_{q}>=\frac{1}{H} \int_{-H}^{0} M_{q}(z) \mathrm{d} z$ and $\frac{1}{H} \int_{-H}^{0} u^{2} \mathrm{~d} z=1=\sum_{q=0}^{+\infty} \frac{1}{H} u_{q}^{2}$.

\subsection{Usual approximation of depth independent barotropic mode}

A usual approximation of the barotropic mode is the following. Considering that $\epsilon=N^{2}(0) H / g$ is small, the surface boundary condition (17) can be approximated by

$$
\frac{\mathrm{d} M_{q}}{\mathrm{~d} z}(0)=0
$$

This approximation corresponds to the one done in rigid lid ocean models where $\frac{\partial \eta}{\partial t}=0$. In this approximation, 0 is an eigenvalue of the Sturm Liouville system (15)-(17) and the approximated modes (denoted here by $\widetilde{M}$ ) satisfy the following properties

$$
\widetilde{M}_{0}(z)=1, \quad \int_{-H}^{0} \widetilde{M}_{q}(z) \mathrm{d} z=0 \text { for } q \geq 1
$$

The corresponding "barotropic" velocity and pressure components are thus obtained by a simple vertical averaging:

$$
\widetilde{u}_{0}(x, t)=\bar{u}(x, t)=\frac{1}{H} \int_{-H}^{0} u(x, z, t) \mathrm{d} z, \quad \widetilde{h}_{0}(x, t)=\frac{1}{\rho_{0} g} \bar{p}(x, t)=\frac{1}{\rho_{0} g H} \int_{-H}^{0} p(x, z, t) \mathrm{d} z
$$

A direct time integration of $\widetilde{h}_{0}(x, t)$ is not possible anymore since (19) is not valid for $\lambda_{0}=0$ (i.e. $c_{0}=+\infty$ ) but the pressure can be decomposed into surface and internal values using

$$
p(x, z, t)=\rho_{0} g \eta(x, t)+p_{h}(x, z, t)
$$


with

$$
\begin{gathered}
p_{h}(x, 0, t)=0 \\
\frac{\partial p_{h}}{\partial z}=-\rho g
\end{gathered}
$$

so that $\widetilde{h}_{0}(x, t)=\eta(x, t)+\frac{1}{\rho_{0} g H} \int_{-H}^{0} p_{h}(x, z, t) \mathrm{d} z$. At the expense of a clear inconsistency with the assumption used to approximate the barotropic mode, the time evolution of the free surface $\eta$ is then given by the surface boundary condition (6):

$$
\frac{\partial \eta}{\partial t}=w(z=0)
$$

and $w$ at the surface is deduced from the vertical integration of the continuity equation $(4)$ to get $w(x, z=$ $0, t)=-H \frac{\partial \bar{u}}{\partial x}(x, t)$. The corresponding barotropic system is thus finally given by:

$$
\left\{\begin{aligned}
\frac{\partial \bar{u}}{\partial t}+g \frac{\partial}{\partial x}\left(\eta+\frac{1}{\rho_{0} g H} \int_{-H}^{0} p_{h}\right) & =0 \\
\frac{\partial \eta}{\partial t}+H \frac{\partial \bar{u}}{\partial x} & =0
\end{aligned}\right.
$$

\subsection{Depth dependent versus depth independent barotropic system}

As shown above, the depth independent approximation replaces the system of type (18)-(19) for $q=0$ (the true barotropic mode) by the sytem (30) which actually includes contribution from all modes. As a matter of fact, in this depth independent approximation, the vertical average of the internal pressure can be decomposed as

$$
\frac{1}{H} \int_{-H}^{0} p_{h} \mathrm{~d} z=\frac{1}{H} \int_{-H}^{0} \sum_{q \geq 0} \rho_{0} g h_{q}\left[M_{q}(z)-M_{q}(0)\right] \mathrm{d} z=\rho_{0} g \sum_{q \geq 0}\left(\frac{1}{H} \int_{-H}^{0} M_{q}(z)-M_{q}(0)\right) h_{q}
$$

In particular, the contribution of the barotropic mode is not zero but proportional to $\left(\frac{1}{H} \int_{-H}^{0} M_{0}(z) \mathrm{d} z-M_{0}(0)\right)$ which, in the case of constant $N$, can be expressed using (23) as

$$
\frac{1}{H} \int_{-H}^{0} M_{0}(z) \mathrm{d} z-M_{0}(0) \approx \frac{\epsilon}{3}
$$

In numerical models using this depth independent approximation, the vertically integrated internal pressure gradient of (30) is put on the right hand side and is held constant during the time integration of the barotropic velocity. This means in particular that the contribution of the barotropic mode, although rapidly changing, is also held constant, which is the main source of instability in this linear framework.

Similarly it can be shown that the approximated baroclinic part $(u-\bar{u})$ has a barotropic component of order $\epsilon^{2}$ and this will have an impact in term of stability (see $\left.\S 4.4\right)$.

\subsection{Illustration of the numerical instability in an idealized test case}

Let us illustrate here the aforementioned instability, using an idealized test case. The linearized primitive equations (2)-(5) are solved on a periodic 2-D $(x, z)$ domain of length $L_{x}=10000 \mathrm{~km}$ with a depth of $H=4 \mathrm{~km}$. The Brünt Vaisala frequency $N$ is taken equal to $10^{-3} \mathrm{~s}^{-1}$ (constant background stratification 
case). The model is initialized with a barotropic field corresponding to a wave travelling to the right $\left(u_{0}-\frac{g}{c_{0}} h_{0}=0\right)$ :

$$
h_{0}(x, t=0)=10 \sin \left(\frac{2 \pi}{L_{x}} x\right) e^{-\left(x-L_{x}\right)^{2} /(2 \Delta)^{2}}, \quad u_{0}(x, t=0)=\frac{g}{c_{0}} h_{0}(x, 0)
$$

with $\Delta=2000 \mathrm{~km}$. The initial velocity, density and free surface fields are then deduced:

$u(x, z, t=0)=u_{0}(x, t=0) M_{0}(z), \quad \rho(x, z, t=0)=-\rho_{0} h_{0}(x, t=0) \frac{\mathrm{d} M_{0}(z)}{\mathrm{d} z}, \quad \eta(x, t=0)=h_{0}(x, t=0) M_{0}(0)$

The initial density field $\rho(x, z, t=0)$ is plotted on Figure 1:

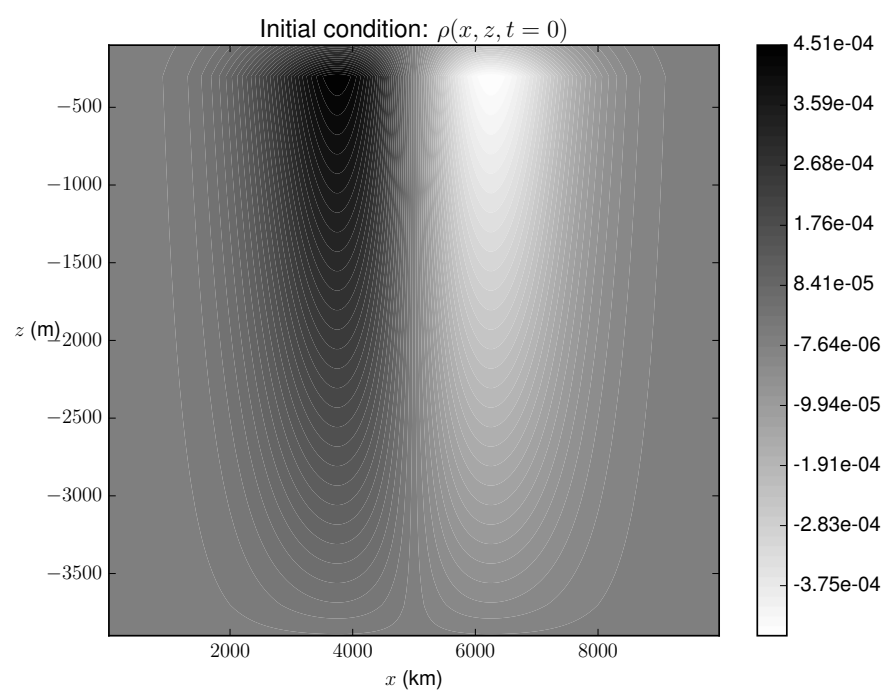

Figure 1: Initial value of the density field

Discretization and numerical schemes. The model uses a horizontally staggered grid (Arakawa $C$-grid) and a Lorenz vertical grid with a geopotential vertical coordinate. It uses second order finite differences. The horizontal and vertical grid resolutions are $\Delta x=50 \mathrm{~km}$ and $\Delta z=50 \mathrm{~m}$. The 3D time integration scheme is the non dissipative Euler Forward Backward scheme. The 2D time integration for the barotropic part of the flow is also based on a non dissipative Forward Backward scheme. The value of the barotropic time steps $\Delta t_{0}$ is chosen so that the $2 \mathrm{D}$ Courant number $\nu_{0}=\frac{c_{0} \Delta t_{0}}{\Delta x}=0.75$. The splitting ratio $N_{\text {split }}=\Delta t / \Delta t_{0}$ is chosen equal to 60 , leading to a value of the 3D Courant number $\nu_{1}=\frac{c_{1} \Delta t}{\Delta x}=0.31$ (this mode splitting strategy will be explained in detail in $§ 3.1)$.

Results. As a diagnostic, we look at the time evolution of the maximum absolute value of the free surface elevation. The result is compared to a reference solution without mode splitting: the 3D equations are integrated with a time step that fulfills the barotropic CFL condition. Figure 2 clearly illustrates the instability, with a growing maximum value of the free surface elevation in the splitting case. 


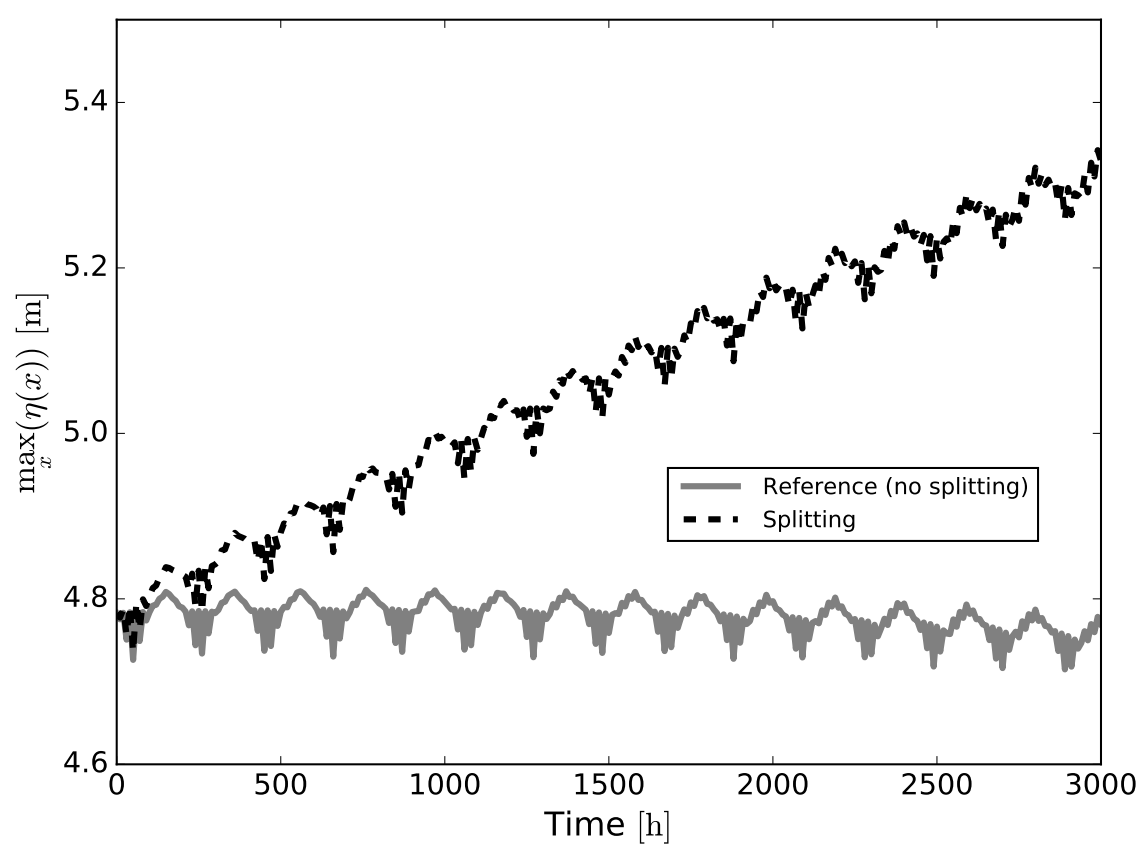

Figure 2: Time evolution of the maximum absolute value of the free surface elevation. Comparaison of the nummerical solution with (dashed line) and without (plain line) splitting.

\section{Formalization of the time integration scheme using normal mode decomposition}

In this section, in order to explore the stability of the mode splitting approach when the depth-averaged approximation of the barotropic mode is made, we will formalize the time integration scheme, using the basis composed of the vertical modes $M_{q}(z)$.

\section{1. $2 D$-3D correction}

Similarly to $\S 2.6$, let $\Delta t$ be the baroclinic time step and $N_{\text {split }}$ the number of barotropic sub-time steps. The barotropic time step is given by $\Delta t_{0}=\frac{\Delta t}{N_{\text {split }}}$.

The stability of the barotropic/baroclinic splitting will be affected by the following key points:

1. the dissipation put in the 2D part, either through averaging filters or through explicit diffusion (see $\S 4.1)$;

2. the computation of the internal pressure gradient;

3. The 3D time integration scheme.

At time step $n$, we assume the consistency conditions:

$$
\bar{u}^{n}=\frac{1}{H} \int_{-H}^{0} u_{3 \mathrm{~d}}^{n} \quad \text { and } \quad p^{n}(z=0)=\rho_{0} g \eta^{n}
$$

An example of a simple time integration algorithm is depicted in Figure 3. 


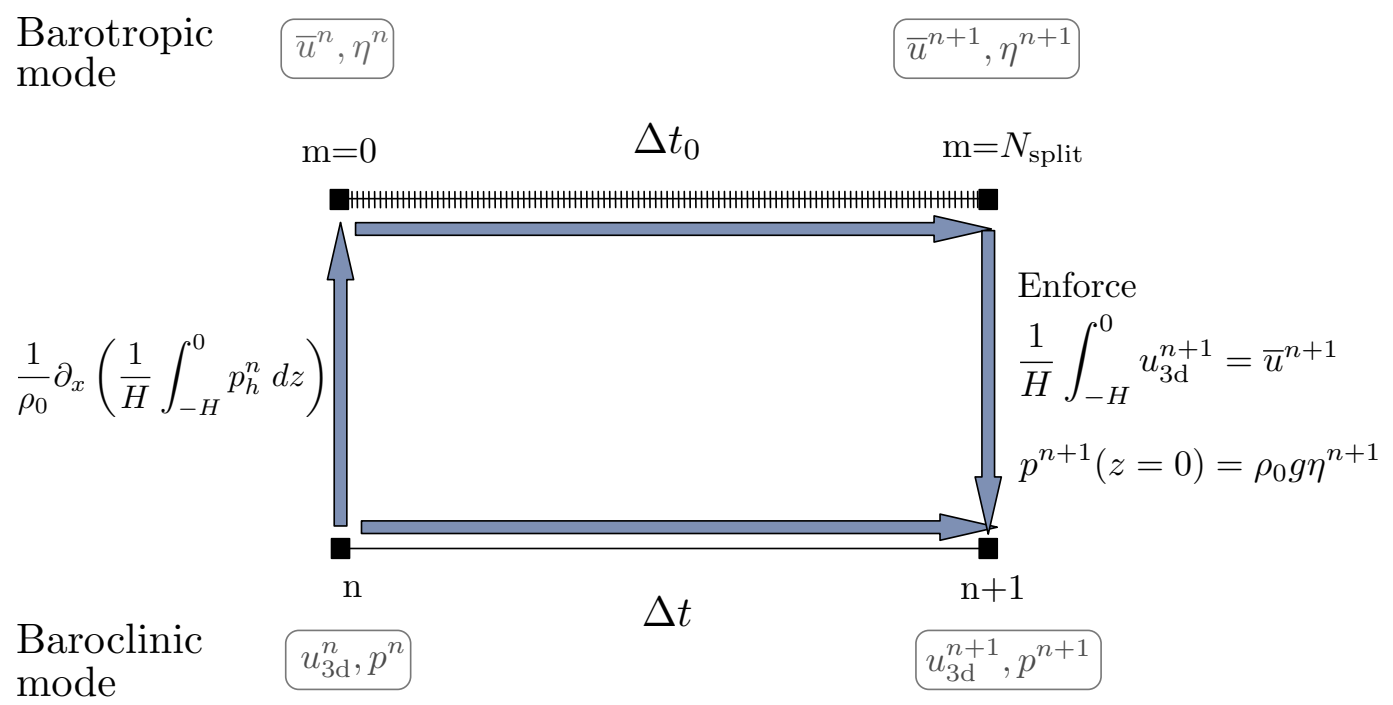

Figure 3: An example of a mode splitting algorithm where the vertically integrated pressure gradient is computed at time $n$

In this simple case, the hydrostatic pressure gradient is computed at time step $n$ (other alternatives will be discussed in $§ 4.4)$ and vertically integrated to provide the right hand side of the barotropic momentum equations which are integrated with the small time step $\Delta t_{0}$. The $3 \mathrm{D}$ equations are themselves integrated with the large time step $\Delta t$.

After the 2D barotropic and 3D baroclinic integrations, the consistency conditions at time step $n+1$ imply two corrections:

1. The first one is to modify the $3 \mathrm{D}$ velocities so that their vertical average matches the barotropic (depth averaged) velocity $\bar{u}^{n+1}$. If $u_{3 \mathrm{~d}}^{n+1}$ and $u_{3 \mathrm{~d}}^{n+1, \mathrm{c}}$ denote the $3 \mathrm{D}$ velocities at time step $n+1$ before and after correction, the correction step reads

$$
u_{3 \mathrm{~d}}^{n+1, \mathrm{c}}=u_{3 \mathrm{~d}}^{n+1}+\left[\bar{u}^{n+1}-\bar{u}_{3 \mathrm{~d}}^{n+1}\right] \quad \text { with } \bar{u}_{3 \mathrm{~d}}^{n+1}=\frac{1}{H} \int_{-H}^{0} u^{n+1} \mathrm{~d} z
$$

In term of projected variables $u_{q}$, this correction can be written as

$$
\begin{aligned}
u_{q}^{n+1, c}=<u_{3 \mathrm{~d}}^{n+1, \mathrm{c}}, M_{q}> & =u_{q}^{n+1}+\left(\frac{1}{H} \int_{-H}^{0} M_{q}(z) \mathrm{d} z\right)\left[\bar{u}^{n+1}-\bar{u}_{3 \mathrm{~d}}^{n+1}\right] \\
& =u_{q}^{n+1}+\alpha_{q}^{2} M_{q}(0)\left[\bar{u}^{n+1}-\bar{u}_{3 \mathrm{~d}}^{n+1}\right]
\end{aligned}
$$

2. In the 2D integration, the free surface evolves and this leads to a modification of the surface pressure gradient $p(z=0)=\rho_{0} g \eta$. This evolution has to be in agreement with the modal decomposition which states that $p(z=0)=\rho_{0} g \sum_{q=0}^{K-1} h_{q} M_{q}(0)$ (note that, once the depth $H$ discretized into $K$ cells, the vertical mode decomposition leads to the barotropic mode and $K-1$ baroclinic modes). This second correction on $h_{q}$ must ensure that

$$
p^{n+1}(z=0)=\rho_{0} g \eta^{n+1}=\rho_{0} g \sum_{q=0}^{K-1} h_{q}^{n+1, c} M_{q}(0)
$$


This correction can be written as:

$$
h_{q}^{n+1, \mathrm{c}}=h_{q}^{n+1}+\alpha_{q}^{4} M_{q}(0)\left(\eta^{n+1}-\eta_{3 \mathrm{~d}}^{n+1}\right), \quad \text { where } \eta_{3 \mathrm{~d}}^{n+1}=p^{n+1}(z=0) /\left(\rho_{0} g\right)=\sum_{j=0}^{K-1} h_{j}^{n+1} M_{j}(0)
$$

Indeed it leads to $\sum_{q} h_{q}^{n+1, \mathrm{c}} M_{q}(0)=\sum_{q} h_{q}^{n+1} M_{q}(0)+\left(\eta^{n+1}-\eta_{3 \mathrm{~d}}^{n+1}\right) \sum_{q} \alpha_{q}^{4} M_{q}(0)^{2}=\eta^{n+1}$ since $\sum \alpha_{q}^{4} M_{q}(0)^{2}=1$ using $(26)-(27)$.

Note that satisfying $(33,34)$ is equivalent to ensuring that the density field (hence the $h_{q}$ components) has to be integrated with a velocity field which has a vertical average that matches the one needed to make the free surface $\eta$ evolve from time step $n$ to time step $n+1$. Indeed if $\overline{\bar{u}}$ satisfies $\frac{\eta^{n+1}-\eta^{n}}{\Delta t}=-\frac{\partial H \overline{\bar{u}}}{\partial x}$, we can write

$$
h_{q}^{n+1}=h_{q}^{n}-\frac{\Delta t}{g \lambda_{q} H} \frac{\partial}{\partial x} H u_{q}^{\star}, \quad h_{q}^{n+1, c}=h_{q}^{n}-\frac{\Delta t}{g \lambda_{q} H} \frac{\partial}{\partial x} H\left(u_{q}^{\star}+\alpha_{q}^{2} M_{q}(0)\left[\overline{\bar{u}}-\overline{u^{\star}}\right]\right)
$$

where $u^{\star}$ is the $3 \mathrm{D}$ velocity field used to integrate the density field (its specific location in time is dependent on the time stepping). We get

$$
\begin{aligned}
h_{q}^{n+1, c}-h_{q}^{n+1} & =\frac{\Delta t}{g \lambda_{q} H} \alpha_{q}^{2} M_{q}(0)\left[\frac{\eta^{n+1}-\eta^{n}}{\Delta t}-\frac{\eta_{3 \mathrm{~d}}^{n+1}-\eta^{n}}{\Delta t}\right] \\
& =\alpha_{q}^{4} M_{q}(0)\left[\eta^{n+1}-\eta_{3 \mathrm{~d}}^{n+1}\right] \operatorname{using} \lambda_{q}=1 /\left(\alpha_{q}^{2} g H\right)
\end{aligned}
$$

which is identical to $(34)$.

In the literature this correction is also known as constancy preservation (e.g. [13, 4]) since, in a free surface nonlinear model, it ensures that an initially constant tracer field will remain constant.

\subsection{Temporal evolution in matrix form}

Let $X_{q}=\left(\begin{array}{c}u_{q} \\ h_{q}\end{array}\right)$ be the vector of velocity and pressure projections onto the mode $M_{q}$. The system (18)-(19) can be written as:

$$
\frac{\partial X_{q}}{\partial t}+A_{q} \frac{\partial X_{q}}{\partial x}=0
$$

with

$$
A_{q}=\left(\begin{array}{cc}
0 & g \\
\frac{c_{q}^{2}}{g} & 0
\end{array}\right)
$$

The corrections (32)-(34) can be formulated as:

$$
X_{q}^{n+1, \mathrm{c}}=X_{q}^{n+1}+C_{q}\left(\begin{array}{c}
\bar{u}^{n+1}-\bar{u}_{3 \mathrm{~d}}^{n+1} \\
\eta^{n+1}-\eta_{3 \mathrm{~d}}^{n+1}
\end{array}\right)
$$

where

$$
C_{q}=\left(\begin{array}{cc}
\alpha_{q}^{2} M_{q}(0) & 0 \\
0 & \alpha_{q}^{4} M_{q}(0)
\end{array}\right)
$$

Since, for baroclinic modes $(q \geq 1), \alpha_{q}=\mathcal{O}(\sqrt{\epsilon})$, Eq. (35) shows that the correction is $\mathcal{O}(\epsilon)$ for velocity components and $\mathcal{O}\left(\epsilon^{2}\right)$ for pressure components. 
The objective is now to express the vector $\left(\begin{array}{c}\bar{u}^{n+1,2 \mathrm{~d}}-\bar{u}^{n+1,3 \mathrm{~d}} \\ \eta^{n+1,2 \mathrm{~d}}-\eta^{n+1,3 \mathrm{~d}}\end{array}\right)$ according to the 2D barotropic integration. Using the fact that the internal pressure gradient is held constant during the barotropic integration, i.e. $\frac{\partial \zeta}{\partial t}=\frac{\partial \eta}{\partial t}$ within a baroclinic time step $\Delta t$, the barotropic system (30) can be rewritten as

$$
\left\{\begin{array}{ll}
\frac{\partial \bar{u}}{\partial t}+g \frac{\partial \zeta}{\partial x} & =0 \\
\frac{\partial \zeta}{\partial t}+\frac{\partial H \bar{u}}{\partial x} & =0
\end{array} \quad \text { on }[n \Delta t,(n+1) \Delta t]\right.
$$

where $\zeta=\eta+\frac{1}{\rho_{0} g} \frac{1}{H} \int_{-H}^{0} p_{h} \mathrm{~d} z$. Using $A^{2 \mathrm{~d}}$ the matrix corresponding to the discrete 2D time stepping algorithm over one barotropic sub-time step, we write

$$
\left(\begin{array}{c}
\bar{u}^{n+1} \\
\zeta^{n+1}
\end{array}\right)=\left[A^{2 \mathrm{~d}}\right]^{N_{\mathrm{split}}}\left(\begin{array}{c}
\bar{u}^{n} \\
\zeta^{n}
\end{array}\right)
$$

where $N_{\text {split }}$ is the number of barotropic sub-time steps.

The evolution of the free surface is thus given by:

$$
\left(\begin{array}{c}
\bar{u}^{n+1} \\
\eta^{n+1}
\end{array}\right)=\left[A^{2 \mathrm{~d}}\right]^{N_{\mathrm{split}}}\left(\begin{array}{c}
\bar{u}^{n} \\
\eta^{n}
\end{array}\right)+\left(\left[A^{2 \mathrm{~d}}\right]^{N_{\mathrm{split}}}-I\right)\left(\begin{array}{c}
0 \\
\frac{1}{\rho_{0} g H} \int_{-H}^{0} p_{h} \mathrm{~d} z
\end{array}\right)
$$

At time step $n$, the $2 \mathrm{D}$ variables $\bar{u}$ and $\eta$ can be expressed as

$$
\bar{u}^{n}=\bar{u}_{3 \mathrm{~d}}^{n}=\sum_{p} \alpha_{p}^{2} M_{p}(0) u_{p}^{n}, \quad \eta^{n}=\eta_{3 \mathrm{~d}}^{n}=\sum_{p} h_{p}^{n} M_{p}(0)
$$

so that

$$
\left(\begin{array}{c}
\bar{u}^{n} \\
\eta^{n}
\end{array}\right)=\sum_{p} V_{p}\left(\begin{array}{c}
u_{p}^{n} \\
h_{p}^{n}
\end{array}\right), \quad \text { with } V_{p}=M_{p}(0)\left(\begin{array}{cc}
\alpha_{p}^{2} & 0 \\
0 & 1
\end{array}\right)
$$

As mentioned before, we assume that the internal pressure gradient is computed at time step $n$ in (38). Using (31), the decomposition of the internal pressure gradient is

$$
\frac{1}{\rho_{0} g} \frac{1}{H} \int_{-H}^{0} p_{h}^{n} \mathrm{~d} z=\sum_{p}\left(\alpha_{p}^{2}-1\right) M_{p}(0) h_{p}^{n},
$$

so that

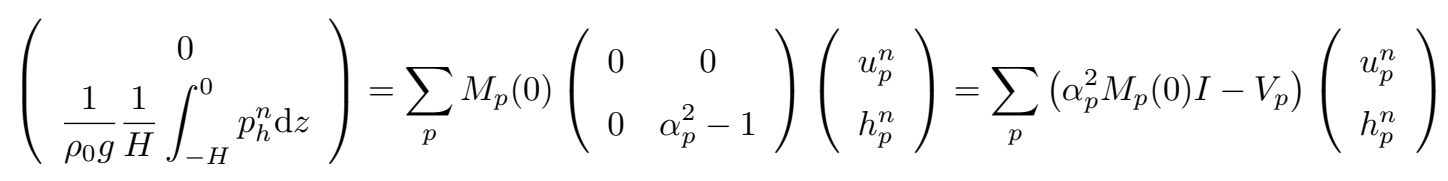

We deduce from (38) :

$$
\begin{aligned}
\left(\begin{array}{c}
\bar{u}^{n+1} \\
\eta^{n+1}
\end{array}\right) & =\sum_{p}\left(\left[A^{2 \mathrm{~d}}\right]^{N_{\mathrm{split}}} V_{p}+\left(\left[A^{2 \mathrm{~d}}\right]^{N_{\mathrm{split}}}-I\right)\left(\alpha_{p}^{2} M_{p}(0) I-V_{p}\right)\right)\left(\begin{array}{c}
u_{p}^{n} \\
h_{p}^{n}
\end{array}\right) \\
& =\sum_{p}\left(V_{p}+\alpha_{p}^{2} M_{p}(0)\left(\left[A^{2 \mathrm{~d}}\right]^{N_{\mathrm{split}}}-I\right)\right)\left(\begin{array}{c}
u_{p}^{n} \\
h_{p}^{n}
\end{array}\right)
\end{aligned}
$$


which we will write as:

$$
\left(\begin{array}{c}
\bar{u}^{n+1} \\
\eta^{n+1}
\end{array}\right)=\sum_{p} V_{p} A_{p}^{2 \mathrm{~d}}\left(\begin{array}{c}
u_{p}^{n} \\
h_{p}^{n}
\end{array}\right)
$$

with

$$
A_{p}^{2 \mathrm{~d}}=I+W_{p}\left(\left[A^{2 \mathrm{~d}}\right]^{N_{\mathrm{split}}}-I\right), \quad W_{p}=\left(\begin{array}{cc}
1 & 0 \\
0 & \alpha_{p}^{2}
\end{array}\right)
$$

$A_{p}^{2 d}$ is the matrix that makes the projections evolve during the barotropic sub-time steps. For the barotropic component $(p=0), \alpha_{0} \approx 1, W_{0} \approx I$ and this matrix is close to $\left[A^{2 \mathrm{~d}}\right]^{N_{\text {split }}}$.

\section{Evolution through the $3 D$ integration and $2 D / 3 D$ correction}

We denote by $A_{p}^{3 \mathrm{~d}}$ the amplification matrix of the 3D baroclinic time step, such that, in a family of one step time integration schemes,

$$
\left(\begin{array}{c}
u_{p}^{n+1} \\
h_{p}^{n+1}
\end{array}\right)=A_{p}^{3 \mathrm{~d}}\left(\begin{array}{c}
u_{p}^{n} \\
h_{p}^{n}
\end{array}\right)
$$

We thus have

$$
\left(\begin{array}{c}
\bar{u}_{3 d}^{n+1} \\
\eta_{3 d}^{n+1}
\end{array}\right)=\sum_{p} V_{p} A_{p}^{3 \mathrm{~d}}\left(\begin{array}{c}
u_{p}^{n} \\
h_{p}^{n}
\end{array}\right)
$$

and

$$
\left(\begin{array}{c}
\bar{u}^{n+1} \\
\eta^{n+1}
\end{array}\right)-\left(\begin{array}{c}
\bar{u}_{3 \mathrm{~d}}^{n+1} \\
\eta_{3 \mathrm{~d}}^{n+1}
\end{array}\right)=\sum_{p} V_{p}\left(A_{p}^{2 \mathrm{~d}}-A_{p}^{3 \mathrm{~d}}\right)\left(\begin{array}{c}
u_{p}^{n} \\
h_{p}^{n}
\end{array}\right) .
$$

Combining (35) and (41), we finally get:

$$
\begin{aligned}
X_{q}^{n+1, c} & =X_{q}^{n+1}+C_{q} \sum_{p} V_{p}\left(A_{p}^{2 \mathrm{~d}}-A_{p}^{3 \mathrm{~d}}\right) X_{p}^{n} \\
& =A_{q}^{3 \mathrm{~d}} X_{q}^{n}+C_{q} \sum_{p} V_{p}\left(A_{p}^{2 \mathrm{~d}}-A_{p}^{3 \mathrm{~d}}\right) X_{p}^{n}
\end{aligned}
$$

\section{Stability analysis}

In $\S 2.6$, we have illustrated the unstable behaviour of the depth-independent approach which is due to the pressure gradient term held constant during the barotropic integration. To counter this instability and the potential other sources of splitting errors, ocean models rely on additional dissipation put on the barotropic mode dynamics. In $\S 4.1$ below, different ways of introducing dissipation are presented, and then a stability analysis is performed in the rest of this section.

\subsection{Discussion on usual filters}

In this subsection, we discuss the two possible ways of introducing dissipation in the barotropic mode before reconciliation with its 3D counterpart: averaging filters and diffusion within the barotropic time stepping. The averaging filters are implemented as

$$
\bar{u}_{\mathrm{F}}^{n+1}=\sum_{m=0}^{N_{\text {filter }}} a_{m} \bar{u}^{m}
$$


where the weights $a_{m}$ are normalized and such that the average is centered at time step $n+1$ (see [3]):

$$
\sum_{m=0}^{N_{\text {filter }}} a_{m}=1, \quad \frac{1}{N_{\text {split }}} \sum_{m=0}^{N_{\text {filter }}} m a_{m}=1
$$

Note that when using averaging filters, in order to be able to center the average at time $n+1$, the window of integration of the barotropic equations has of course to be extended beyond $n+1$. These extra computations can have a significant impact on the total computational cost, especially for parallel simulations where the ratio of computation to communication is low in comparison to the $3 \mathrm{D}$ parts (i.e. the $2 \mathrm{D}$ integration becomes the limiting factor in the scaling efficiency of the code).

Let present now two usual averaging filters (flat filter and cosine filter), as well as a common time stepping algorithm (forward-backward scheme) that can also be modified to act as a filter. The weights $a_{m}$ of the two filters are displayed in Figure 4.

Let $\mu_{0}=k c_{0} \Delta t, k$ being the wavenumber of a Fourier component of the numerical solution, and let $d_{0}\left(\mu_{0}\right)$

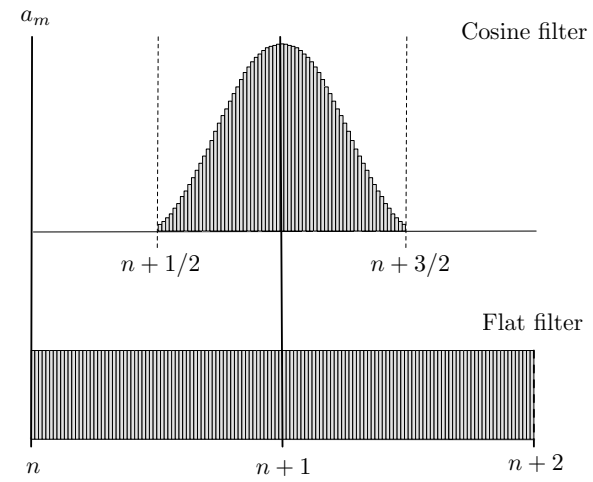

Figure 4: The weights shape of two averaging filters: cosine filter (top) and flat filter (bottom).

be the damping factor associated with these filters. Expressions of $d_{0}$ can be easily obtained:

- Flat filter of size $N_{\text {filter }}=2 N_{\text {split }}: a_{m}=1 /\left(2 N_{\text {split }}+1\right)$

$$
\begin{aligned}
d_{0}^{\text {Flat }} e^{i \mu_{0}} & =\frac{1}{2 N_{\text {split }}+1} \sum_{m=0}^{2 N_{\text {split }}} e^{i m \frac{\mu_{0}}{N_{\text {split }}}} \\
& =\frac{1}{2 N_{\text {split }}+1} e^{i \mu_{0}} \frac{\sin \left(\left(2 N_{\text {split }}+1\right) \mu_{0} / 2 N_{\text {split }}\right)}{\sin \left(\mu_{0} / 2 N_{\text {split }}\right)} \\
& \approx \frac{\sin \mu_{0}}{\mu_{0}} e^{i \mu_{0}} \quad \text { for large } N_{\text {split }}
\end{aligned}
$$

The development of $d_{0}^{\text {Flat }}$ at low frequencies leads to

$$
d_{0}^{\text {Flat }}\left(\mu_{0}\right) \simeq 1-\frac{1}{6} \mu_{0}^{2}
$$

- Cosine filter of size $N_{\text {filter }}=3 N_{\text {split }} / 2: a_{m}=0$ for $m<N_{\text {split } / 2}, a_{m}=\frac{1}{N_{\text {split }}}\left[1+\cos 2 \pi \frac{m}{N_{\text {split }}}\right]$ for $N_{\text {split }} / 2 \leq$ 


$$
\begin{aligned}
m \leq 3 N_{\text {split }} / 2 & \\
d_{0}^{\cos } e^{i \mu_{0}}=\frac{1}{N_{\text {split }}} \sum_{m=N_{\text {split }} / 2}^{m=3 N_{\text {split }} / 2}\left[1+\cos 2 \pi \frac{m}{N_{\text {split }}}\right] e^{i m \frac{\mu_{0}}{N_{\text {split }}}} & =\frac{2}{N_{\text {split }} \frac{\left(\sin \frac{\pi}{N_{\text {split }}}\right)^{2} \sin \frac{\mu_{0}}{2}}{2 N_{\text {split }}}\left(\cos \frac{\mu_{0}}{N_{\text {split }}}-\cos \frac{2 \pi}{N_{\text {split }}}\right)} e^{i \mu_{0}} \\
& \approx \frac{\sin \left(\mu_{0} / 2\right)}{\frac{\mu_{0}}{2}\left(1-\frac{\mu_{0}^{2}}{4 \pi^{2}}\right)} \quad \text { for large } N_{\text {split }}
\end{aligned}
$$

The development of $d_{0}^{\text {cos }}$ at low frequencies leads to

$$
d_{0}^{\cos }\left(\mu_{0}\right) \simeq 1-\left(\frac{1}{24}-\frac{1}{4 \pi^{2}}\right) \mu_{0}^{2}
$$

- A dissipative Forward Backward (FB) scheme. Let consider the following forward backward scheme, which is a diffusive $2 \mathrm{D}$ time stepping :

$$
\begin{cases}\bar{u}^{m+1}=\bar{u}^{m}-\Delta t_{0} g \partial_{x} \zeta^{m} & \text { for } 0 \leq m \leq N_{\text {split }}-1 \\ \zeta^{m+1}=\zeta^{m}-\Delta t_{0} \partial_{x}\left[(1+\theta) \bar{u}^{m+1}-\theta \bar{u}^{m}\right] H & \end{cases}
$$

where $\Delta t_{0}=\frac{\Delta t}{N_{\text {split }}}$ is the barotropic time step. It is well known that the scheme (45) is neutral for $\theta=0$ and is dissipative for $\theta>0$. The corresponding amplification factor over $N_{\text {split }}$ time steps reads:

$$
d_{0}^{\mathrm{FB}}\left(\mu_{0}\right)=\left(\sqrt{1-\theta\left(\frac{\mu_{0}}{N_{\text {split }}}\right)^{2}}\right)^{N_{\text {split }}}
$$

The development of $d_{0}$ at low frequencies leads to

$$
d_{0}^{\mathrm{FB}}\left(\mu_{0}\right) \simeq 1-\gamma \mu_{0}^{2} \quad \text { with } \gamma=\frac{\theta}{2 N_{\text {split }}}
$$

To illustrate the damping effect of these filters, we fixed some parameters. The ratio $N_{\text {split }}$ is naturally close to $\frac{c_{0}}{c_{1}}\left(\approx \frac{\pi}{N} \sqrt{\frac{g}{H}}\right)$, its actual value depending on the stability conditions of the 2D and 3D time stepping algorithms. Typical maximum values of $\mu_{0}=k c_{0} \Delta t$ will also be close to $\frac{c_{0}}{c_{1}}$.

For a value of $H=4000 \mathrm{~m}$, we consider the two values of the Brunt Vaisala frequency $N$ given in Table 1. The numerical values of the $2 \mathrm{D}$ FB parameter $\theta=\frac{\epsilon N_{\text {split }}}{6}$ are also given since, in the next subsection, these values (which imply $\gamma=\frac{\epsilon}{12}$ in (46)) will be proved to guarantee the stability of the splitting.

\begin{tabular}{|c|c|c|c|c|}
\hline$N$ & $\frac{c_{0}}{c_{1}}$ & $\epsilon=\frac{N^{2} H}{g}$ & $N_{\text {split }}$ & $\theta=\frac{\epsilon N_{\text {split }}}{6}$ \\
\hline $10^{-2}$ & 15 & 0.041 & 20 & 0.14 \\
$10^{-3}$ & 155 & 0.00041 & 200 & 0.014 \\
\hline
\end{tabular}

Table 1: Set of parameters $N$ and corresponding values of $\frac{c_{0}}{c_{1}}, \epsilon, N_{\text {split }}, \theta$ for $H=4000 \mathrm{~m}$

Figure 5 shows the damping factors $\left|d_{0}\left(\mu_{0}\right)\right|$ for the two averaging filters and for the dissipative FB scheme. For this FB scheme, $\theta$ is given by $\theta=0.14$ which corresponds to the strong stratification case $N=10^{-2}$. Note that we choose this value of $\theta$ even in the weakly stratified case $N=10^{-3}$ since in practice, in the numerical model, $\theta$ will imposed by the maximum value of $N$ over the whole domain. This figure illustrates the strong differences among all these filters. 

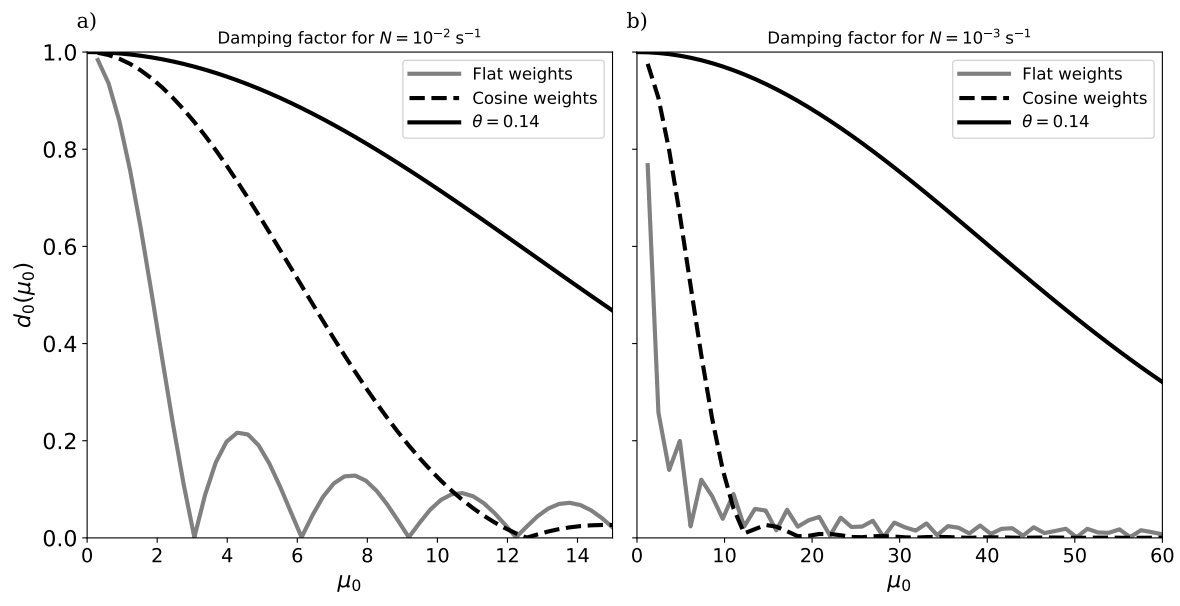

Figure 5: Damping factor $\left|d_{0}\left(\mu_{0}\right)\right|$ as a function of $\mu_{0}$ for the flat (thin plain line) and cosine (dashed line) averaging filters and for the dissipative Forward Backward (FB, thick plain line) scheme (with $\theta=0.14$ ) for $N=10^{-2}, N_{\text {split }}=20$ (left), $N=10^{-3}, N_{\text {split }}=200$ (right). $\mu_{0}$ is in the range $\left[0, \frac{c_{0}}{c_{1}}\right]$. The right plot is however limited to $\mu_{0} \leq 60$.

\subsection{Stability of the barotropic mode integration}

We first study the stability of the $2 \mathrm{D}$ integration alone with respect to the barotropic variables $\left(u_{0}, h_{0}\right)$. According to (42), the evolution of the barotropic components is given by $A_{0}^{3 \mathrm{~d}}+C_{0} V_{0}\left(A_{0}^{2 \mathrm{~d}}-A_{0}^{3 \mathrm{~d}}\right)=A_{0}^{2 \mathrm{~d}}+$ $\left(C_{0} V_{0}-I\right)\left(A_{0}^{2 \mathrm{~d}}-A_{0}^{3 \mathrm{~d}}\right)$. Since $C_{0} V_{0}-I=\left(\alpha_{0}^{4} M_{0}^{2}(0)-1\right) I$ is, using (23), of the order of $\epsilon^{2}$, the last term will be neglected in this subsection and the whole evolution of the barotropic mode is approximated by $A_{0}^{2 \mathrm{~d}}$. This term, and thus the impact of the three dimensional time stepping included in $A_{0}^{3 \mathrm{~d}}$, will be taken into account in subsection 4.3.

The question is here is to determine the minimum amount of diffusion to add to the $2 \mathrm{D}$ integration to compensate for the fact that the depth integrated vertical pressure gradient is held constant. Let's suppose that the $2 \mathrm{D}$ system (37) has been integrated without dispersion errors and with a damping factor $d_{0} . A^{2 \mathrm{~d}}$ corresponds to a shallow water system with a propagation speed of $\sqrt{g H}$ (or $c_{0} / \alpha_{0}$ ) leading (in Fourier space) to:

$$
\left[A^{2 \mathrm{~d}}\right]^{N_{\mathrm{split}}}=P_{0}^{\mathrm{rl}}\left(\begin{array}{cc}
d_{0} e^{-i \mu_{0} / \alpha_{0}} & 0 \\
0 & d_{0} e^{i \mu_{0} / \alpha_{0}}
\end{array}\right)\left(P_{0}^{\mathrm{rl}}\right)^{-1}, \text { with }\left(P_{0}^{\mathrm{rl})^{-1}}=\left(\begin{array}{cc}
1 & \alpha_{0} \frac{g}{c_{0}} \\
1 & -\alpha_{0} \frac{g}{c_{0}}
\end{array}\right)\right.
$$

where $\mu_{0}=k c_{0} \Delta t\left(\mu_{0} / \alpha_{0}=k \sqrt{g H} \Delta t\right), k$ being the wavenumber. $A_{0}^{2 d}$ is given by (40) for $p=0$. Except within small intervals around $\cos \mu_{0} / \alpha_{0}=-1$ (or equivalently $k \sqrt{g H} \Delta t=(2 p+1) \pi$ with $p$ an integer), the matrix $A_{0}^{2 d}$ has two complex conjugate eigenvalues $\left(\lambda_{0}, \overline{\lambda_{0}}\right)$ and its determinant has the following simple expression:

$$
\operatorname{det}\left(A_{0}^{2 d}\right)=\left|\lambda_{0}\right|^{2}=d_{0}\left(\alpha_{0}^{2} d_{0}-\left(\alpha_{0}^{2}-1\right) \cos \mu_{0} / \alpha_{0}\right)
$$

Let us first study low frequencies (or large horizontal scales) $\mu_{0} \ll 1$ and assume that at those scales $d_{0}\left(\mu_{0}\right)$ can be developed as $d_{0}=1-\gamma \mu_{0}^{2}$ (second order damping). A second order Taylor expansion leads to

$$
\left|\lambda_{0}\right|^{2}=1+\left(\frac{1}{2} \frac{\alpha_{0}^{2}-1}{\alpha_{0}^{2}}-\gamma\left(1+\alpha_{0}^{2}\right)\right) \mu_{0}^{2}+\mathcal{O}\left(\mu_{0}^{4}\right)
$$


Since $\alpha_{0}>1$ (see 23,25), this shows that if $\gamma=0$ (no damping) the model is unstable. This instability is tracked back to the computation of the pressure gradient at time step $n$ (Euler Forward scheme). The minimum value of $\gamma$ that leads to $\left|\lambda_{0}\right| \leq 1$ is given by

$$
\gamma \geq \frac{\alpha_{0}^{2}-1}{2 \alpha_{0}^{2}\left(\alpha_{0}^{2}+1\right)}
$$

and (assuming constant $N$ ) using expression (23) of $\alpha_{0}$ and a first order expansion in $\epsilon$ we get:

$$
\gamma \geq \frac{\epsilon}{12}
$$

Eq. 49 is a necessary condition for the stability of the mode splitting approach (under the condition of a pressure gradient computed at time step $n$ ). It implies that at large scales the filter has to correspond to a second order filter with a minimum value given by (49).

At smaller scales (i.e. for larger values of $\mu_{0}$ ), the maximum of the eigenvalues module is attained at points where $\cos \left(\mu_{0} / \alpha_{0}\right)=-1$. At these points $A_{0}^{2 d}$ is given by

$$
A_{0}^{2 d}\left(\cos \left(\mu_{0} / \alpha_{0}\right)=-1\right)=\left(\begin{array}{cc}
-d_{0} & 0 \\
0 & 1-\alpha_{0}^{2}\left(1+d_{0}\right)
\end{array}\right) .
$$

The maximum of eigenvalues module is then given by $\left|\lambda_{0}\right|=\left|1-\alpha_{0}^{2}\left(1+d_{0}\right)\right| \approx\left(d_{0}+\frac{1+d_{0}}{3} \epsilon\right)$. (Due to this, all the following figures representing the magnitudes of the barotropic modes will contain special points (at $\left.\mu_{0}=(2 p+1) \alpha_{0} \pi\right)$ where the two barotropic modes have distinct magnitudes.) Figure 6 illustrates the barotropic mode amplification (i.e. maximum eigenvalue of $A_{0}^{2 \mathrm{~d}}$ ) for values of $N=10^{-2}, H=4000$ ( $\epsilon=0.041)$ both without damping $\left(d_{0}=1\right)$ and when the filters described in $\S 4.1$ are activated. All filters are able to stabilize the barotropic mode integration (both at large and small scales) ${ }^{1}$.
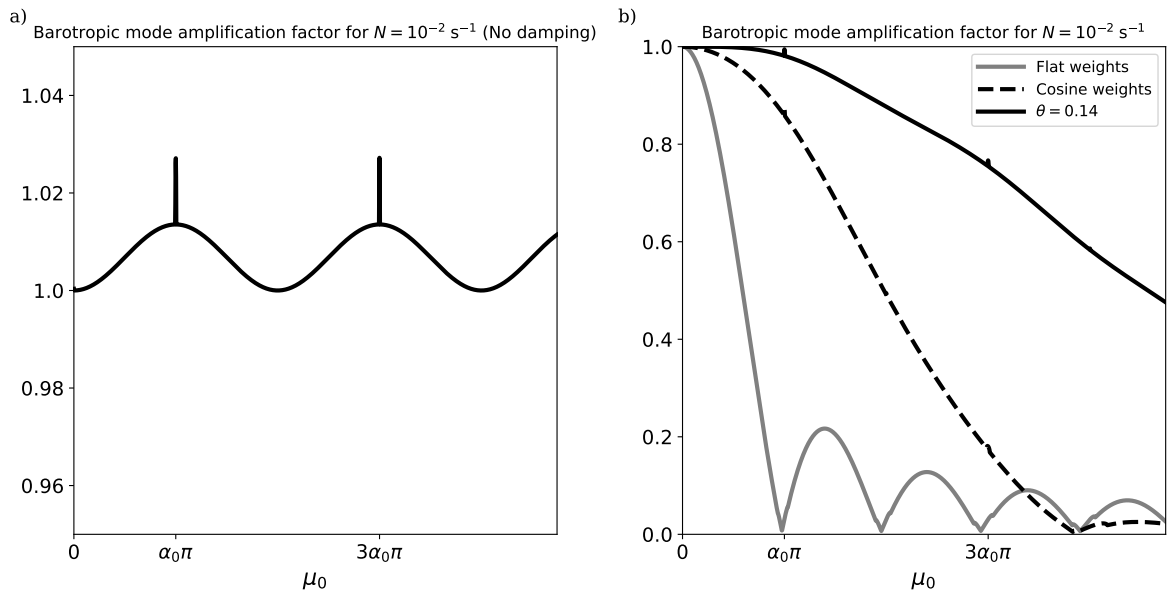

Figure 6: Barotropic mode amplification for $N=10^{-2}, H=4000$. a) No damping, b) with damping according to different filters (plain line: Flat filter; dashed line: Cosine filter; Thick plain line: FB scheme with $\theta=0.14$ ).

\footnotetext{
${ }^{1}$ For the Forward Backward dissipative time stepping, $\left|\lambda_{0}\left(\mu_{0}=\alpha_{0} \pi\right)\right| \approx 1-\epsilon\left(\pi^{2} / 12-2 / 3\right)<1$
} 


\subsection{Addition of the first baroclinic mode}

We here add one baroclinic mode in the analysis, mainly to look at the damping of this first baroclinic mode associated with the 2D filtering. This will also allow to look at the impact of the 3D time stepping algorithm and at the impact of the centering of the vertically integrated pressure gradient. Since for higher baroclinic modes, the corrections will be smaller, it is expected that the stability of the first baroclinic mode ensures the stability of higher order baroclinic modes, and of the total integration. This is what has been observed in real simulations.

The system coming from (42) can be written as :

$$
X^{n+1, \text { corrected }}=G X^{n}
$$

where $X$ is truncated to the first two modes: $X=\left(X_{0}, X_{1}\right)^{T}$ and with

$$
G=\left(\begin{array}{cc}
\left.C_{0} V_{0} A_{0}^{2 \mathrm{~d}}+\left(I-C_{0} V_{0}\right) A_{0}^{3 d}\right) & C_{0} V_{1}\left(A_{1}^{2 \mathrm{~d}}-A_{1}^{3 \mathrm{~d}}\right) \\
C_{1} V_{0}\left(A_{0}^{2 \mathrm{~d}}-A_{0}^{3 \mathrm{~d}}\right) & A_{1}^{3 \mathrm{~d}}+C_{1} V_{1}\left(A_{1}^{2 \mathrm{~d}}-A_{1}^{3 \mathrm{~d}}\right)
\end{array}\right)
$$

$A_{0}^{2 d}$ is the same as in the previous section (i.e. without dispersion errors). Going on with the family of one step, forward in time, algorithms, the three dimensional fields are integrated with a simple forward backward scheme that reads as follows:

$$
\begin{aligned}
& u_{q}^{n+1}=u_{q}^{n}-\Delta \operatorname{tg} \frac{\partial h_{q}^{n}}{\partial x} \\
& h_{q}^{n+1}=h_{q}^{n}-\Delta t \frac{c_{q}^{2}}{g} \frac{\partial u_{q}^{n+1}}{\partial x}
\end{aligned}
$$

In terms of the original variables $(u, w, \rho)$, this scheme corresponds to:

$$
\begin{aligned}
& \frac{\partial p^{n}}{\partial z}=-g \rho^{n} \\
& u^{n+1}=u^{n}-\Delta t \frac{1}{\rho_{0}} \frac{\partial p^{n}}{\partial x} \\
& \frac{\partial u^{n+1}}{\partial x}+\frac{\partial w^{n+1}}{\partial z}=0 \\
& \rho^{n+1}=\rho^{n}-\Delta t w^{n+1} \frac{\mathrm{d} \bar{\rho}}{\partial z}
\end{aligned}
$$

It is well known that the Forward Backward scheme (51) is neutral when it is stable (i.e. under condition $c_{q} k \Delta t \leq 2$ ). It will typically be unstable for the barotropic mode (i.e. $A_{0}^{3 d}$ has eigenvalues with modulus larger than 1). The amplifications of the barotropic and baroclinic modes are plotted on figure (7) in the case of no damping $\left(d_{0}=1\right)$. The barotropic mode is unstable at all scales while the baroclinic modes have small instabilities at small scales. 


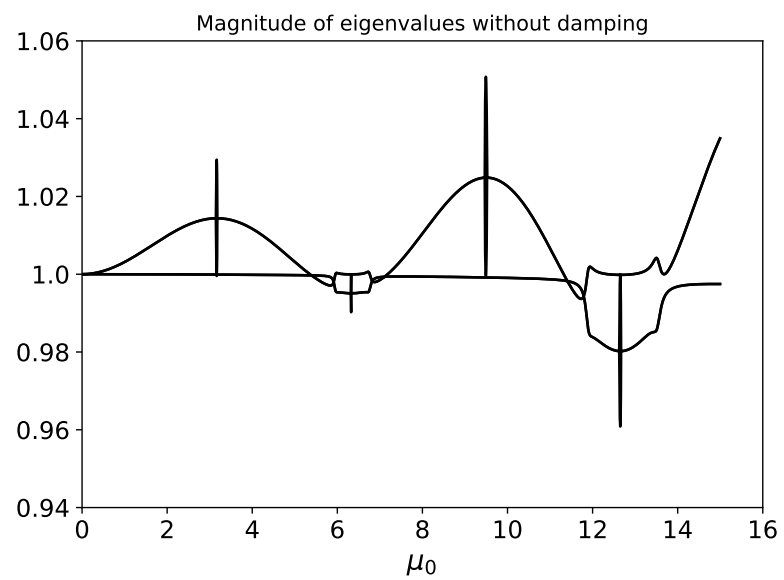

Figure 7: Magnitude of eigenvalues without damping for $N=10^{-2}\left(N_{\text {split }}=20\right)$. Four curves are superimposed corresponding to the left and right going barotropic and baroclinic waves.

Figure (8) shows the same amplification factors with added filtering for the case $N=10^{-2}$. The filtering is added either using a flat filter, a cosine filter or using a 2D Forward backward diffusive time stepping (with $\theta=0.14$ as above). All filters are able to stabilize the integration and as expected the dissipative Forward Backward scheme is less diffusive than the averaging filters.
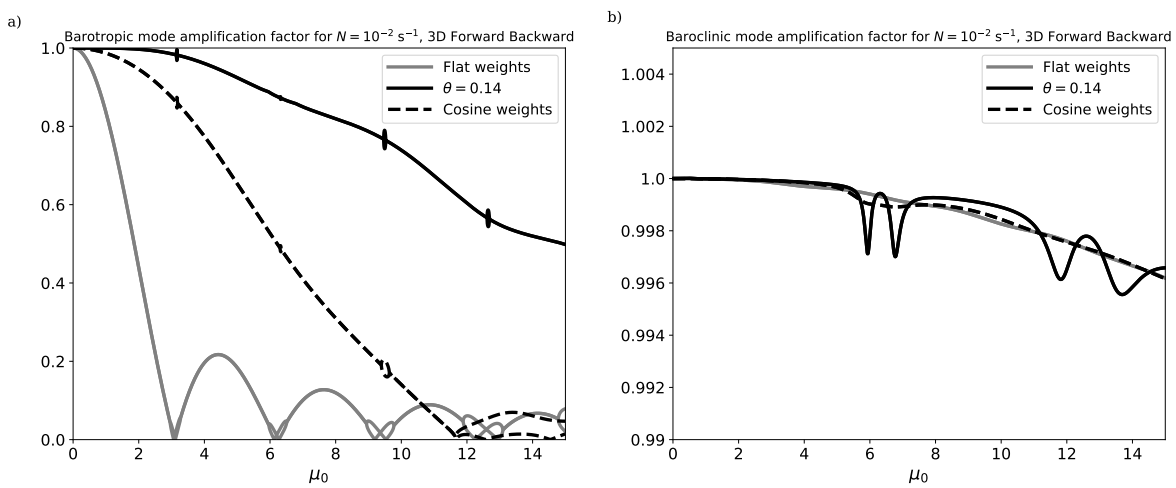

Figure 8: Barotropic (left) and baroclinic (right) modes amplification for $N=10^{-2}$ and $N_{\text {split }}=20$. Black line: FB scheme, black dashed line: cosine filter, grey line: flat filter.

\subsection{Alternate temporal discretizations}

The general framework developed in $\S 3.2$ has been applied in $\S 4.2$ and $\S 4.3$ with two discretization choices: the vertical integral of the internal pressure gradient is computed using the density field (hence the $h_{q} \mathrm{~s}$ ) at time step $n$ and the 3D equations are integrated using a Forward Backward scheme with a specific order (see Eqs (51)): the velocity field is integrated before the density field. Here we investigate other alternatives. The order of integration in the $3 \mathrm{D}$ equations is specified by the parameter $\beta$ in the following equations.

$$
\begin{aligned}
& u_{q}^{n+1}=u_{q}^{n}-\Delta \operatorname{tg} \frac{\partial}{\partial x}\left(\beta h_{q}^{n}+(1-\beta) h_{q}^{n+1}\right) \\
& h_{q}^{n+1}=h_{q}^{n}-\Delta t \frac{c_{q}^{2}}{g} \frac{\partial}{\partial x}\left((1-\beta) u_{q}^{n}+\beta u_{q}^{n+1}\right)
\end{aligned}
$$


$\beta$ can be either 0 or 1 , in order to keep the time integration explicit. $\beta=1$ leads to the previous ForwardBackward scheme while $\beta=0$ reverses the integration order.

We also consider additional ways of computing the vertical integral of the internal pressure gradient which forces the 2D barotropic equations. The corresponding density field can also be chosen as a weighted average of the density field at time $n$ and $n+1$ (deduced from the time integration of the 3D equations):

$$
h_{q}^{\star}=\gamma h_{q}^{n}+(1-\gamma) h_{q}^{n+1}
$$

or extrapolated from past values:

$$
h_{q}^{\star}=\frac{3}{2} h_{q}^{n}-\frac{1}{2} h_{q}^{n-1}
$$

$h_{q}^{\star}$ is then used to provide the vertical integral of the pressure gradient (i.e. $h_{q}^{n}$ is replaced by $h_{q}^{\star}$ in Eq. (39). The choice made in the previous subsections was $\beta=1$ in (52) and the formulation (53) with $\gamma=1$.

Note that one interest of choosing formulation (53) with $\gamma=1 / 2$ or the extrapolation (54) is that it leads to a second order accurate time integration of the depth-dependent barotropic components $\left(u_{0}, h_{0}\right)$.

Figure (9) shows the magnitude of the corresponding eigenvalues (barotropic and first baroclinic modes). We dot not detail the computations. These eigenvalues have been numerically computed using a simple extension of the previous framework:

- The formulation (53) with $\gamma \neq 1$ makes the $A_{q}^{2 \mathrm{~d}}$ matrices dependent on the $A_{q}^{3 \mathrm{~d}}$ matrices.

- The extrapolation (54) introduces another level $(n-1)$ of time variables that has to be taken into account in the framework. The eigenvalues associated with the computational modes have been removed from Figure 9 since they do not affect the stability. 


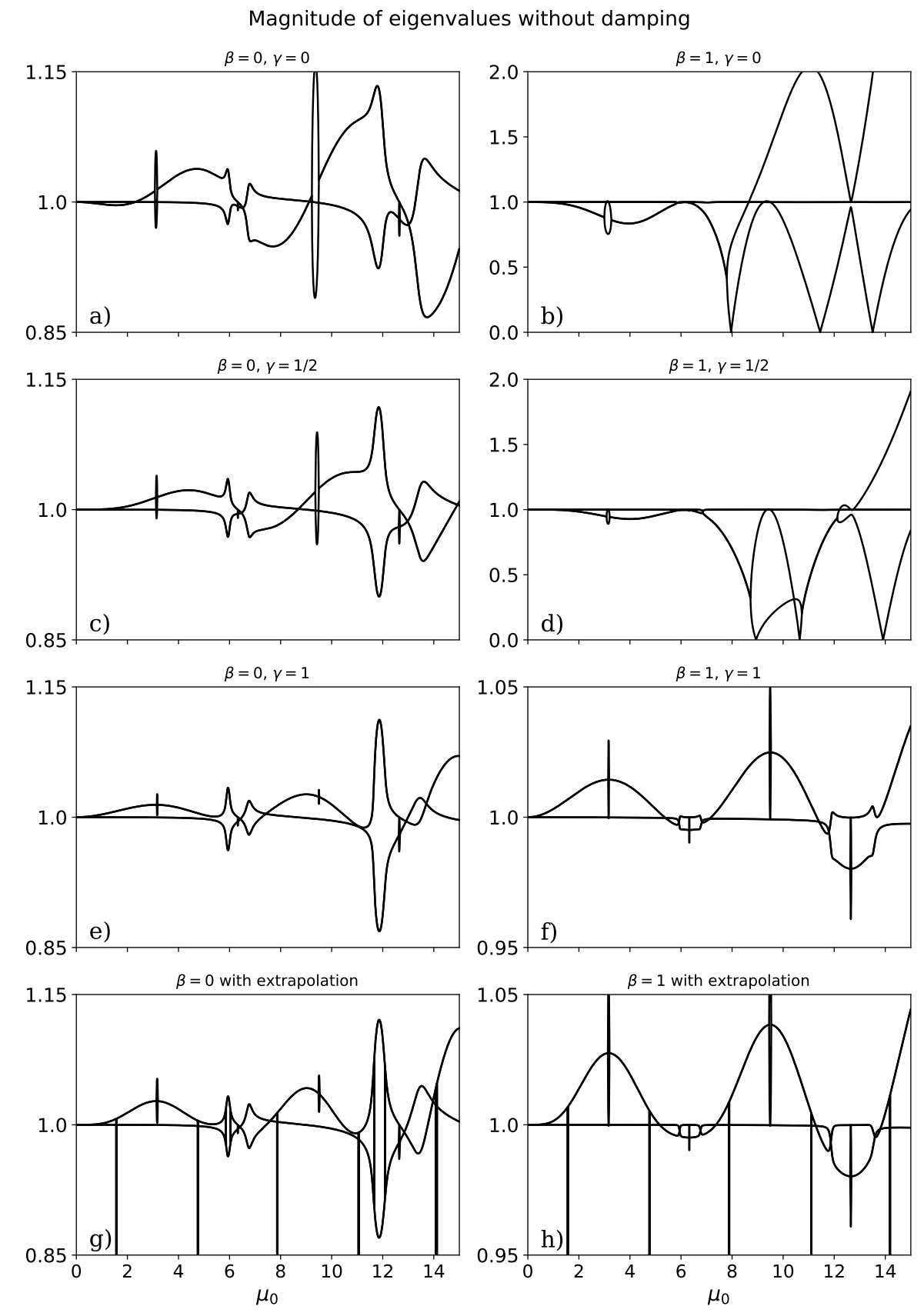

Figure 9: Magnitude of eigenvalues for several choices of $\beta, \gamma$ and for $N=10^{-2}\left(N_{\text {split }}=20\right)$. Note that the vertical axes differ among the plots.

Comments on Figure 9:

1. The weighted formulation $(53)$ is stable at large scales $\left(\mu_{0} \ll 1\right)$ for $\gamma<\frac{1}{2}$ (Fig. 9a,b) as can be seen in the Taylor development of the amplifcation factor:

$$
\left|\lambda_{0}\right|=1+\frac{\epsilon}{12}(-1+2 \gamma) \mu_{0}^{2}+\mathcal{O}\left(\mu_{0}^{4}\right)
$$

For $\gamma=\frac{1}{2}$, we obtain:

$$
\left|\lambda_{0}\right|=1+\frac{\epsilon}{144}(1+6(1-2 \beta) \beta) \mu_{0}^{4}+\mathcal{O}\left(\mu_{0}^{6}\right)
$$


and the formulation is stable at large scales for $\beta=1$ (Fig. 9d) unstable for $\beta=0$ (Fig. 9c)

limit of the 3D integration scheme.

\section{Numerical experiments}

In this section, we will illustrate the theoretical study both on the simple idealized test case, introduced in $\S 2.6$, that relies on the linearized (around a state at rest) primitive equations $(2,3,4,5)$, and then on bottom topography effects. 


\subsection{Idealized test case}

This idealized test case has been described in $§ 2.6$. We present here the numerical results with respect to the chosen filtering techniques. As a diagnostic of the induced damping of the different filtering techniques we again look at the evolution of the maximum of the free surface elevation. Figure 10 shows the results obtained with filtering techniques presented in $§ 4.1$. In addition to the flat and cosine filters described above, we add results obtained with the second order power law averaging filter implemented in the ROMS ([3]). This scheme is known to be less dissipative than the previously presented averaging filters. In particular, it is second order accurate. Note that we did not include this filter in the preceding sections since the discrete expression of the weights is not explicit (see [3]) and only the asymptotic expression of the amplification factor (i.e. for large $N_{\text {split }}$ ) is known.

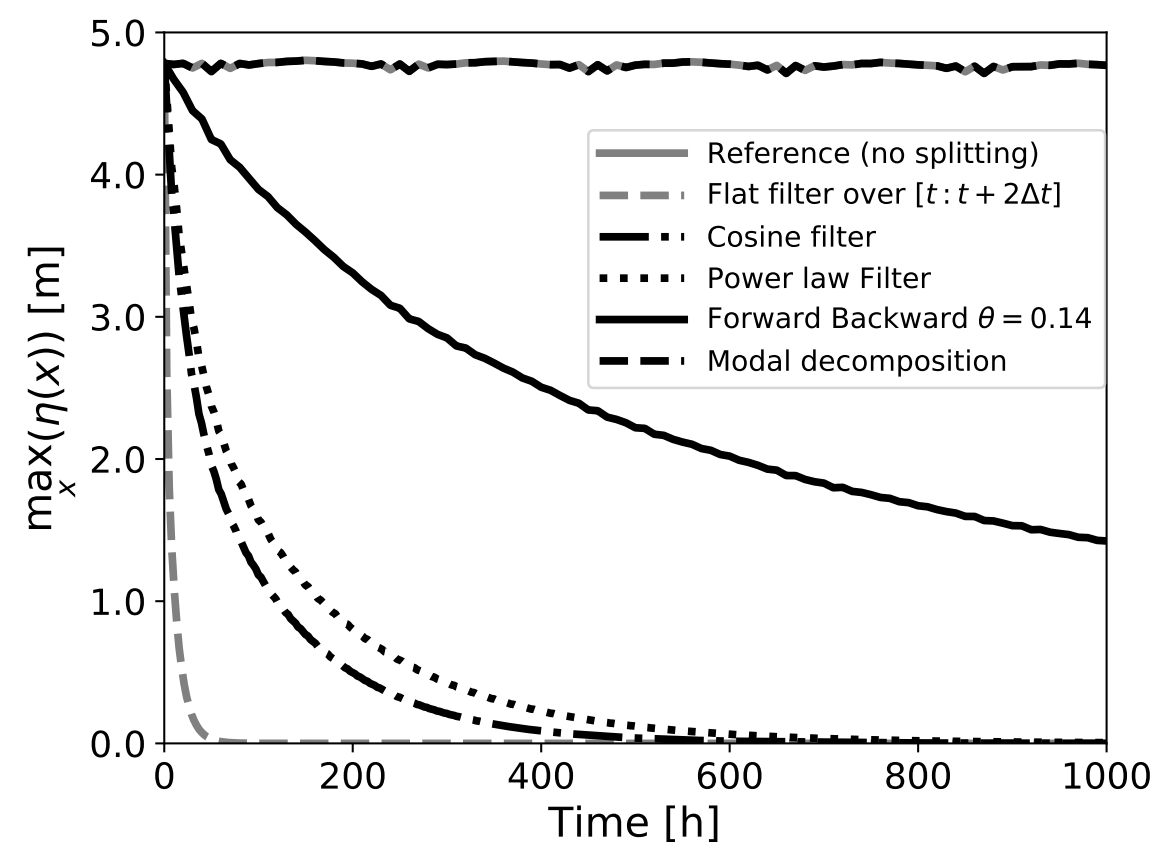

Figure 10: Time evolution of the maximum absolute value of the free surface elevation. Comparaison of usual filters against a reference solution without splitting.

The amount of dissipation varies quite strongly between the different averaging filters and, as expected, the use of the second order power law filter reduces the damping of the free surface elevation. Two other numerical solutions are also presented in Figure 10: the first one corresponds to the use of a diffusive 2D time stepping using a forward backward scheme with $\theta=0.14$, and the second one is based on the use of the true depth-dependent barotropic mode in the mode-splitting separation (see Appendix A for the details of the implementation) without any filtering. The use of a dissipative 2D time stepping is stable and introduces much less damping than the averaging filters. Since there is no damping, the solution obtained with the true barotropic mode exactly matches the reference solution. 


\subsection{Internal tide test case}

We here study the nonlinear internal tide generation by topography. The test case is the same as in [14]. The domain is $2 \mathrm{D} x-z$ with a length of $L=4800 \mathrm{~km}$. The bathymetry is given by $H(x)=$ $5000-1000 e^{-\left(\frac{x-L / 2}{15000}\right)^{2}}$. The initial density field is horizontally homogeneous with a constant vertical gradient given by a Brunt Vaisala frequency $N=1.4 * 10^{-3} \mathrm{~s}^{-1}$. Initial velocities are zero and an initial free surface elevation gradient is prescribed by $\eta(x, t=0)=\frac{x-L / 2}{L / 2}$. The left and right boundaries are closed. The domain geometry leads to the generation of a barotropic wave with a $12 \mathrm{~h}$-period. The total barotropic mechanical energy defined by

$$
E(t)=E_{c}(t)+E_{p}(t)=\int_{0}^{L} \rho_{0} \frac{1}{2}(H+\eta(x, t)) \bar{u}(x, t)^{2}+\int_{0}^{L} \rho_{0} \frac{1}{2} g \eta(x, t)^{2}
$$

During the time integration, part of the barotropic energy will be converted into baroclinic energy while part of the barotropic energy will be dissipated.

\subsubsection{The ROMS model}

We used here the Regional Oceanic Modelling System (ROMS) [3] in its ROMS_AGRIF version (see [15] for differences between the ROMS branches). The model solves the nonlinear hydrostatic primitive equations written in a terrain following vertical coordinate. The time stepping algorithms of the ROMS are as follows: the three dimensional time stepping is a third order predictor corrector time step (Leap Frog - Adams Moulton) while the two dimensional time stepping is a third order generalized forward backward scheme [3]. The internal pressure gradient is extrapolated at time $n+1 / 2$ using a third order Adams Bashforth extrapolation. The model uses third order upstream biased advection schemes for momentum and tracers. The original 2D time stepping has been modified in order to enable the addition of a laplacian diffusion as in $§ 4.1$. The modification of the original scheme is described in Appendix B.

\subsubsection{Numerical settings and results}

The grid resolution is $\Delta x=3 \mathrm{~km}$ in the horizontal, while there are 30 sigma levels in the vertical. The propagation speed of the barotropic mode is $c_{0}=\sqrt{g H} \approx 222 \mathrm{~m} . \mathrm{s}^{-1}$, while the speed of the first internal mode is well approximated by $c_{1}=\frac{N H}{\pi} \approx 2.25 \mathrm{~m} . \mathrm{s}^{-1}$. The value of the small parameter $\epsilon=\frac{N^{2} H}{g}$ is approximately equal to $10^{-3}$. The dissipation of the barotropic mechanical energy is closely linked to the different filters used to prevent the barotropic mode instability. The amount of required diffusion is essentially a function of the baroclinic/barotropic time steps ratio $N_{\text {split }}$. When the baroclinic time step $\Delta t$ is decreased, $N_{\text {split }}$ can be decreased in a proportional way ( $\Delta t_{0}$ keeps the same value) and the amount of diffusion, both via averaging filters or through a $2 \mathrm{D}$ dissipative time stepping will also decrease. What is important to look at is thus the sensitivity of the numerical solution to the splitting ratio $N_{\text {split }}$.

The maximum baroclinic time step allowed by the ROMS time and space baroclinic discretizations is approximatively $\Delta t=960 \mathrm{~s}$. For this maximum value of $\Delta t$, a splitting ratio of $N_{\text {split }}=112\left(\Delta t_{0}=\Delta t / N_{\text {split }} \approx 8.5 \mathrm{~s}\right)$ is required to maintain the stability of the barotropic integration. In the following experiments, the sensitivity study is done for two others values of $\Delta t: \Delta t=240 \mathrm{~s}, \Delta t=60 \mathrm{~s}$ and corresponding splitting ratio $N_{\text {split }}=28, N_{\text {split }}=7$ (the barotropic time step $\Delta t_{0}$ is identical in the three experiments). Solutions are 
given for the power law averaging filter (which is the least dissipative filter among those presented previously) and the 2D dissipative time stepping with second order diffusion. Even if the model is stable with smaller values of $\theta$, we maintain here a diffusion parameter equal to $\theta=0.14^{2}$ which is the (safe) value used in the code for realistic applications. Figure 11 shows the time evolution of the barotropic mechanical energy for the three different choices of $\Delta t$.
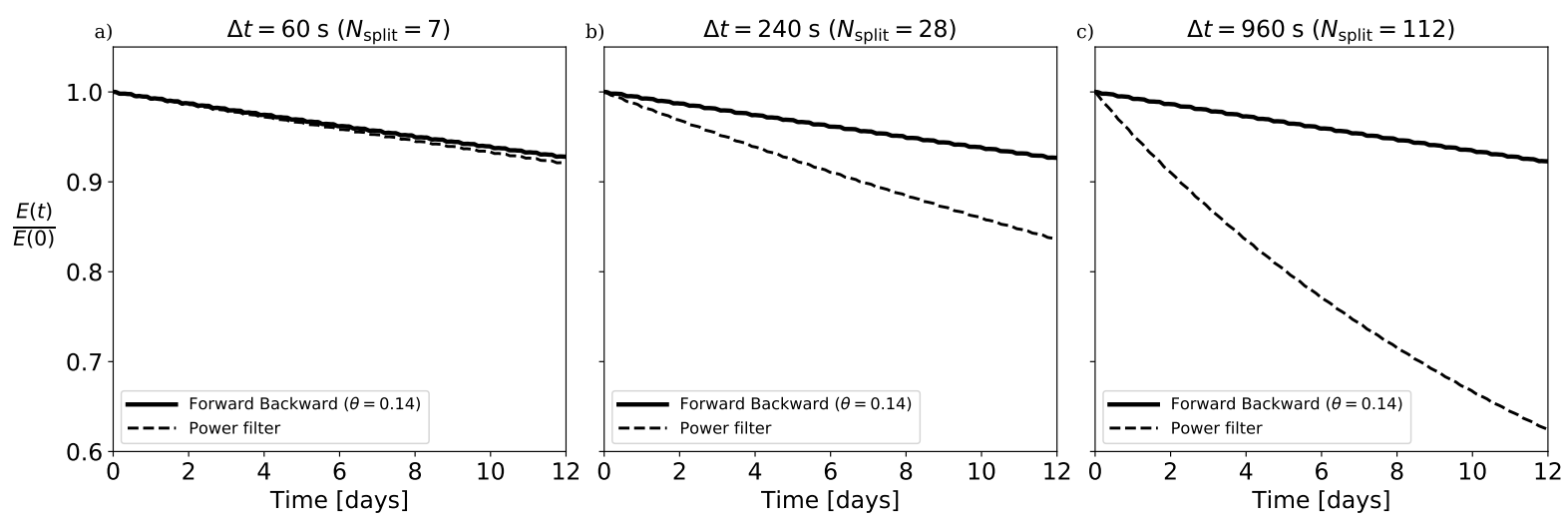

Figure 11: Time evolution of the barotropic mechanical energy $\frac{E(t)}{E(0)}$ for three different baroclinic time step $\Delta t$ and for different filters: Power filter and a Forward Backward diffusive time stepping.

The numerical solution obtained with the averaging filter (power law) is highly sensitive to the choice of the baroclinic time step. With a small time step $(\Delta t=60 \mathrm{~s})$, the solutions obtained with the averaging filter and the forward backward scheme are close, and the decrease of the barotropic mechanical energy is essentially due to conversion to baroclinic energy. But when the time step is increased (hence the splitting ratio $N_{\text {split }}$ accordingly), the dissipation added by the averaging filter is clearly visible. Results (shown on Figure 12) of simulations with a flat bottom configuration (and thus no conversion to baroclinic energy) confirm that the barotropic mechanical energy is almost constant with the forward backward scheme and decreases similarly to Figure 11 with the averaging filter.

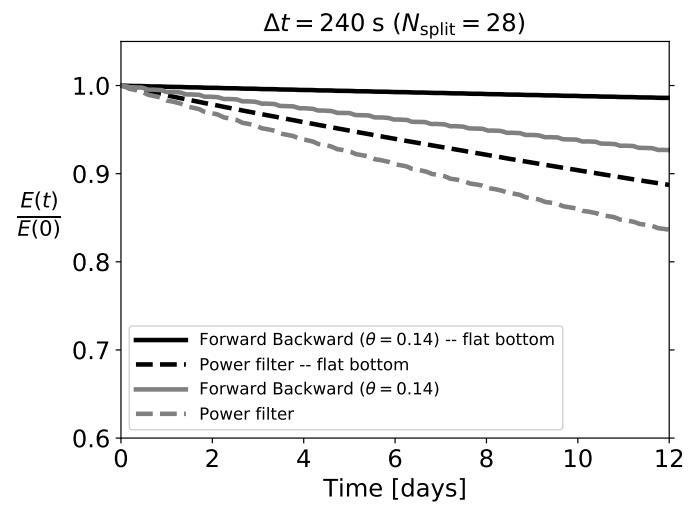

Figure 12: Time evolution of the barotropic mechanical energy $\frac{E(t)}{E(0)}$ for different filters: Power filter and a Forward Backward diffusive time stepping. Difference between flat bottom and non flat bottom experiments.

\footnotetext{
${ }^{2} \theta=0.14$ corresponds to a value of $\alpha=\theta / 2=0.07$ in the scheme presented in Appendix B.
} 
On the computational point of view, as mentioned in $\S 4.1$, simulations with averaging filters require a window of integration of the barotropic equations beyond $t+\Delta t$ in order for the average to be centered at time $t+\Delta t$. For the power law filter, the barotropic model is integrated between approximatively $t$ and $t+3 \Delta t / 2$. The corresponding $2 \mathrm{D}$ simulations are thus 1.5 times more expensive than the ones with the diffusive forward backward scheme. In the configuration studied here, this represents an increase of $10 \%$ of the total computational cost.

\section{Conclusion}

Barotropic/baroclinic mode splitting for free surface ocean models remains an issue since it can require a large amount of non-physical diffusion to achieve a stable integration of the split equations. In this paper, we introduce a framework for the stability analysis of the splitting technique. It is based on a decomposition that uses the true (depth-dependent) barotropic mode, as opposed to the traditional depth-independent assumption adopted in realistic oceanic models, which requires extra sources of diffusion to maintain stability (either through time-filters or dissipative integration schemes). Our study reveals that the amount of diffusion induced by classical averaging filters is much larger than needed to compensate for the inexact mode splitting (i.e. under the depth-independent assumption). We thus favor the use of slightly dissipative 2D time stepping algorithms. Moreover, our stability analysis allows to quantify the minimum amount of required diffusion necessary to counteract mode splitting instabilities due to the depth-independent barotropic mode approximation. Numerical experiments were done here in a very simplified model where all the assumptions of the normal mode decomposition are valid. We have however also run several realistic experiments using the ROMS model. Adding an amount of Laplacian diffusion to the 2D barotropic time stepping algorithm, corresponding to the theoretical study presented in this paper, and removing the existing averaging filter, has led to long terms stable runs. This option is now the default choice in the ROMS_AGRIF model. Moreover, using 2D dissipative time stepping algorithms has several additional advantages over the averaging filters. First the barotropic integration stops at time step $n+1$ and does not require additional time steps like in averaging filters. This lowers the computational cost, especially on parallel computers where the $2 \mathrm{D}$ integration is the less scalable part of the numerical model. It also allows to obtain a continuous free surface elevation. The formulation of a mode splitting technique that uses the depth-dependent barotropic mode has been introduced. In this formulation, the barotropic component of both velocities and density are integrated with small time steps. Several developments are still needed to be able to use this formulation in a realistic context (e.g. non flat bottom) where the normal mode decomposition is not valid and possibly where the density itself is not integrated but diagnosed from temperature and salinity. However, despite these approximations, it is hoped that this formulation will enable a reduction in the amount of numerical diffusion necessary to maintain stability of the mode splitting approach.

The present study has been made assuming a geopotential $(z)$ vertical coordinates system. It could be interesting to perform a similar exercice using different vertical coordinates system, for example using isopycnal vertical coordinates where the barotropic mode is usually defined as a density-weighted vertical average. 


\section{Acknowledgements}

E. Blayo, J. Demange, L. Debreu, and P. Marchesiello were funded by the ANR through contract ANR-11-

MONU-005 (COMODO). C. Eldred was funded by the ANR through contract ANR-14-CE23-0010 (HEAT).

F. Lemarié acknowledges the support of the French LEFE program through project CHRONOS.

[1] J. K. Dukowicz, R. D. Smith, Implicit free-surface method for the bryan-cox-semtner ocean model, Journal of Geophysical Research: Oceans 99 (C4) (1994) 7991-8014. doi:10.1029/93JC03455.

URL http://dx.doi.org/10.1029/93JC03455

[2] P. D. Killworth, D. J. Webb, D. Stainforth, S. M. Paterson, The development of a free-surface bryancox-semtner ocean model, Journal of Physical Oceanography 21 (9) (1991) 1333-1348. doi:10.1175/ 1520-0485 (1991) 021<1333: TDOAFS >2 . 0. CO; 2 .

URL http://dx.doi.org/10.1175/1520-0485(1991)021<1333:TDOAFS>2 . 0.C0;2

[3] A. F. Shchepetkin, J. C. McWilliams, The regional oceanic modeling system (roms): a split-explicit, free-surface, topography-following-coordinate oceanic model, Ocean Modelling 9 (4) (2005) 347 - 404. doi:http://dx.doi.org/10.1016/j.ocemod.2004.08.002.

URL http://www.sciencedirect.com/science/article/pii/S1463500304000484

[4] A. F. Shchepetkin, J. C. McWilliams, Computational kernel algorithms for fine-scale, multiprocess, longtime oceanic simulations, in: P. Ciarlet (Ed.), Handbook of Numerical Analysis, Vol. 14 of Handbook of Numerical Analysis, Elsevier, 2009, pp. 121 -183. doi:http://dx.doi.org/10.1016/S1570-8659(08) 01202-0.

URL http://www.sciencedirect.com/science/article/pii/S1570865908012020

[5] Y. Morel, R. Baraille, A. Pichon, Time splitting and linear stability of the slow part of the barotropic component, Ocean Modelling 23 (3-4) (2008) 73 - 81. doi:http://dx.doi.org/10.1016/j.ocemod. 2008.04 .001$.

URL http://www.sciencedirect.com/science/article/pii/S1463500308000474

[6] B. Nadiga, M. Hecht, L. Margolin, P. Smolarkiewicz, On simulating flows with multiple time scales using a method of averages, Theoretical and Computational Fluid Dynamics 9 (3-4) (1997) 281-292. doi:10.1007/s001620050045.

URL http://dx.doi.org/10.1007/s001620050045

[7] R. Hallberg, Stable split time stepping schemes for large-scale ocean modeling, Journal of Computational Physics 135 (1) (1997) 54 -65. doi:http://dx.doi.org/10.1006/jcph.1997.5734.

URL http://www.sciencedirect.com/science/article/pii/S002199919795734X

[8] R. L. Higdon, R. A. de Szoeke, Barotropic-baroclinic time splitting for ocean circulation modeling, Journal of Computational Physics 135 (1) (1997) 30 - 53. doi:http://dx.doi.org/10.1006/jcph. 1997.5733.

URL http://www.sciencedirect.com/science/article/pii/S0021999197957338 
[9] V. Kamenkovich, D. Nechaev, On the time-splitting scheme used in the princeton ocean model, Journal of Computational Physics 228 (8) (2009) 2874 - 2905. doi : http://dx.doi.org/10.1016/j.jcp. 2008.

[10] P. K. Kundu, I. M. Cohen, Fluid Mechanics, Second Edition, Academic Press, 2002.

[11] E. Blayo, L. Debreu, Revisiting open boundary conditions from the point of view of characteristic variables, Ocean Modelling 9 (3) (2005) 231 - 252. doi:http://dx.doi.org/10.1016/j.ocemod. 2004.07 .001$.

URL http://www.sciencedirect.com/science/article/pii/S1463500304000447

[12] J. K. Dukowicz, Structure of the barotropic mode in layered ocean models, Ocean Modelling 11 (1-2) (2006) 49 -68. doi:http://dx.doi.org/10.1016/j.ocemod.2004.11.005.

URL http://www.sciencedirect.com/science/article/pii/S146350030400099X

[13] S. M. Griffies, R. C. Pacanowski, M. Schmidt, V. Balaji, Tracer conservation with an explicit free surface method for z-coordinate ocean models, Monthly Weather Review 129 (5) (2001) 1081-1098. doi : 10.1175/1520-0493(2001) 129<1081:TCWAEF>2.0.C0;2.

URL http://dx.doi.org/10.1175/1520-0493(2001)129<1081:TCWAEF>2.0.CO;2

[14] P. Marsaleix, F. Auclair, J. W. Floor, M. J. Herrmann, C. Estournel, I. Pairaud, C. Ulses, Energy conservation issues in sigma-coordinate free-surface ocean models, Ocean Modelling 20 (1) (2008) 61 89. doi:http://dx.doi.org/10.1016/j.ocemod.2007.07.005.

URL http://www.sciencedirect.com/science/article/pii/S1463500307000984

[15] A. F. Shchepetkin, J. C. McWilliams, Correction and commentary for "ocean forecasting in terrainfollowing coordinates: Formulation and skill assessment of the regional ocean modeling system" by haidvogel et al., j. comp. phys. 227, pp. 3595-3624, Journal of Computational Physics 228 (24) (2009) 8985 - 9000. doi:http://dx.doi.org/10.1016/j.jcp.2009.09.002.

URL http://www.sciencedirect.com/science/article/pii/S0021999109004872

[16] A. Arakawa, C. S. Konor, Vertical differencing of the primitive equations based on the charney-phillips grid in hybrid sigma-p vertical coordinates, Monthly weather review 124 (3) (1996) 511-528, eng.

[17] D. Holdaway, J. Thuburn, N. Wood, Comparison of lorenz and charney-phillips vertical discretisations for dynamics-boundary layer coupling. part i: Steady states, Quarterly Journal of the Royal Meteorological Society 139 (673) (2013) 1073-1086. doi:10.1002/qj.2016.

URL http://dx.doi.org/10.1002/qj. 2016 


\section{splitting}

The integration of the barotropic part is given by eqs $(18,19)$ for $q=0$

$$
\frac{\partial u_{0}}{\partial t}+g \frac{\partial h_{0}}{\partial x}=0, \quad \frac{\partial h_{0}}{\partial t}+\frac{c_{0}^{2}}{g} \frac{\partial u_{0}}{\partial x}=0
$$

For the practical implementation, we reformulate this equation in term of $u_{0}, \eta, \rho_{b}$ where

$$
\rho_{b}=\frac{1}{H} \int_{-H}^{0} \rho(x, z, t) N^{-2}(z) \frac{\mathrm{d} M_{0}(z)}{\mathrm{d} z} \mathrm{~d} z
$$

$\rho_{b}$ is the barotropic component of the density field and is zero when the barotropic mode is assumed to be depth-independent. One of the reason of introducing $\rho_{b}$ and $\eta$ is to able to have access to the free surface elevation during the barotropic integration. It may be useful for example if the free surface is used in the boundary conditions. But there is a more fundamental reason. If $h_{0}$ is computed inside the barotropic integration, then at the end of this integration the correction is naturally applied to the barotropic component of the pressure field. However when discretized on a vertical Lorenz grid, the presence of a computational mode $([16],[17])$ prevents the determination of a unique correction on the density field (which is what is needed at the end) from a correction on the pressure field.

From the decomposition of $\rho(13)$, we can prove that:

$$
h_{0}=\frac{M_{0}(0)}{\lambda_{0} g H} \eta-\frac{1}{\lambda_{0}} \frac{\rho_{b}}{\rho_{0}}
$$

Note that is this expression $\frac{M_{0}(0)}{\lambda_{0} g H}$ is equal to the vertical integral of the barotropic mode $\int_{-H}^{0} M_{0}(z) \mathrm{d} z$. Integrating (A.2) in (A.1) leads to

$$
\frac{\partial u_{0}}{\partial t}+\frac{\partial}{\partial x}\left[\frac{M_{0}(0)}{\lambda_{0} H} \eta-\frac{g}{\lambda_{0} \rho_{0}} \rho_{b}\right]=0, \quad \frac{\partial \rho_{b}}{\partial t}-\frac{\rho_{0} M_{0}(0)}{g H} \frac{\partial \eta}{\partial t}-\frac{\rho_{0}}{g} \frac{\partial u_{0}}{\partial x}=0
$$

The time evolution of $\eta$ is as usual given by divergence of the depth integrated transport:

$$
\frac{\partial \eta}{\partial t}+\frac{\partial H \bar{u}}{\partial x}=0
$$

where $\bar{u}$ is expressed as a function of $u_{0}$ and the truly baroclinic part:

$$
\bar{u}(x, t)=\frac{1}{H} \int_{-H}^{0} u(x, z, t)=\frac{1}{H} \int_{-H}^{0}\left[u_{0}(x, t) M_{0}(z)+u^{\prime}(x, z, t)\right]=\frac{M_{0}(0)}{\lambda_{0} g H} u_{0}(x, t)+\frac{1}{H} \int_{-H}^{0} u^{\prime}(x, z, t)
$$

$\frac{1}{H} \int_{-H}^{0} u^{\prime}(x, z, t)$ contains only baroclinic modes and thus is the slow part of the depth averaged velocity. In practice, this term is computed at the beginning of the barotropic integration and is held constant over the barotropic time steps (even if it could also be extrapolated). The final form of the barotropic equations is:

$$
\left\{\begin{array}{l}
\frac{\partial u_{0}}{\partial t}+\frac{\partial}{\partial x}\left[\frac{M_{0}(0)}{\lambda_{0} H} \eta-\frac{g}{\lambda_{0} \rho_{0}} \rho_{b}\right]=0 \\
\frac{\partial \rho_{b}}{\partial t}+\frac{\rho_{0} M_{0}(0)}{g H} \frac{\partial H \bar{u}}{\partial x}-\frac{\rho_{0}}{g} \frac{\partial u_{0}}{\partial x}=0 \\
\bar{u}=\frac{M_{0}(0)}{\lambda_{0} g H} u_{0}+\frac{1}{H} \int_{-H}^{0} u^{\prime}(x, z, t) \\
\frac{\partial \eta}{\partial t}+\frac{\partial H \bar{u}}{\partial x}=0
\end{array}\right.
$$


and we recall that when a vertically constant barotropic mode is assumed, the barotropic system is:

$$
\left\{\begin{aligned}
\frac{\partial \bar{u}}{\partial t}+g \frac{\partial \eta}{\partial x} & =-\frac{1}{\rho_{0}} \frac{\partial}{\partial x} \frac{1}{H} \int_{-H}^{0} p_{h} d z \\
\frac{\partial \bar{\eta}}{\partial t}+\frac{\partial H \bar{u}}{\partial x} & =0
\end{aligned}\right.
$$

Appendix A.1. Correction of $3 d$ variables

The correction step, that makes the barotropic mode coincide with the 3D part has been explained in (3.1) for the case of depth-independent barotropic mode. In the depth-dependent case, it includes in addition a correction of the density field:

$$
\left\{\begin{array}{l}
\frac{1}{H} \int_{-H}^{0} u^{n+1, c} M_{0}=u_{0}^{n+1} \\
\frac{1}{H} \int_{-H}^{0} \rho^{n+1, c} N^{-2} \frac{\mathrm{d} M_{0}(z)}{\mathrm{d} z}=\rho_{b}^{n+1} \\
p^{n+1}(0)=\rho_{0} g \eta^{n+1}
\end{array}\right.
$$

The velocity correction is done as follows

$$
u^{n+1, c}=u^{n+1}+\left[u_{0}^{n+1}-\frac{1}{H} \int_{-H}^{0} u^{n+1} M_{0}(z) \mathrm{d} z\right] M_{0}(z)
$$

while the density correction is:

$$
\rho^{n+1, c}=\rho^{n+1}+\frac{1}{\lambda_{0}-\frac{M_{0}(0)^{2}}{g H}}\left[\rho_{b}^{n+1}-\frac{1}{H} \int_{-H}^{0} \rho^{n+1} N^{-2} \frac{\mathrm{d} M_{0}(z)}{\mathrm{d} z}\right] \frac{\mathrm{d} M_{0}(z)}{\mathrm{d} z}
$$

\section{Appendix B. ROMS generalized forward backward scheme}

The generalized forward backward scheme (AB3- and AM4- like) time step writes:

$$
\begin{aligned}
\zeta^{n+1} & =\zeta^{n}-i \mu\left[\left(\frac{3}{2}+\beta\right) u^{n}-\left(\frac{1}{2}+2 \beta\right) u^{n-1}+\beta u^{n-2}\right] \\
u^{n+1} & =u^{n}-i \mu\left[\delta \zeta^{n+1}+(1-\delta-\gamma-\epsilon) \zeta^{n}+\gamma \zeta^{n-1}+\epsilon \zeta^{n-2}\right]
\end{aligned}
$$

The characteristic polynomial of (B.1) is equal to

$$
P(\lambda)=\frac{1}{2}\left(2(-1+\lambda)^{2} \lambda^{4}+\left(\epsilon+\gamma \lambda-(-1+\delta+\epsilon+\gamma) \lambda^{2}+\delta \lambda^{3}\right)\left(2 \beta(-1+\lambda)^{2}+\lambda(-1+3 \lambda)\right) \mu^{2}\right)
$$

The amplification factors of (B.1) can be obtained by solving the characteristic equation $P(\lambda)=0$. We approximate the amplification of the physical mode of (B.1) by computing a perturbation of the true solution $\lambda=e^{i \mu}$. So we look for $\varepsilon \ll 1$ such that for $\mathcal{P}\left(\lambda_{\varepsilon}\right)=0$ where $\lambda_{\varepsilon}=e^{i \mu}(1+\varepsilon)$ where $\mathcal{P}$ is a Taylor expansion around $\varepsilon=0, \mu=0$. Doing this expansion at first order in $\varepsilon$ and fifth order in $\mu$ results in an analytic form of $\lambda_{\varepsilon}$ (computed by Mathematica). Second order expansion of the modulus of $\lambda_{\varepsilon}$ leads to

$$
\left|\lambda_{\varepsilon}\right|=1+\left(\frac{1}{4}-\frac{\delta}{2}+\epsilon+\frac{\gamma}{2}\right) \mu^{2}+\mathcal{O}\left(\mu^{4}\right)
$$

Requiring the time step to be second order dissipative $\left(\left|\lambda_{\varepsilon}\right|=1-\alpha \mu^{2}+\mathcal{O}\left(\mu^{4}\right)\right)$ leads to :

$$
\delta=\frac{1}{2}+2 \epsilon+\gamma+2 \alpha
$$


We then require that the time step has a third order phase error which leads to the additional constraint given by :

$$
\gamma=\frac{1}{3}-\beta-3 \epsilon-2 \alpha^{2}
$$

Note that by setting $\alpha=0$ in (B.2) and (B.3), we recover the conditions $(2.52,2.57)$ of (Schept2005) for third order accuracy of the time step.

As in (Schept2005), the value of $\beta$ is fixed to 0.281105 which gives the largest stability limit for advection (in case of a non linear system). Once $\alpha$ is specified by the required amount of second order damping of external gravity waves, we have a one parameter $(\epsilon)$ optimization problem for the maximization of the stability range. The optimization is performed under the constraint of monotonic damping of the resulting time step. Figure (B.13) shows the optimum value of $\epsilon$ for varying $\alpha$ in the range [0;0.05].
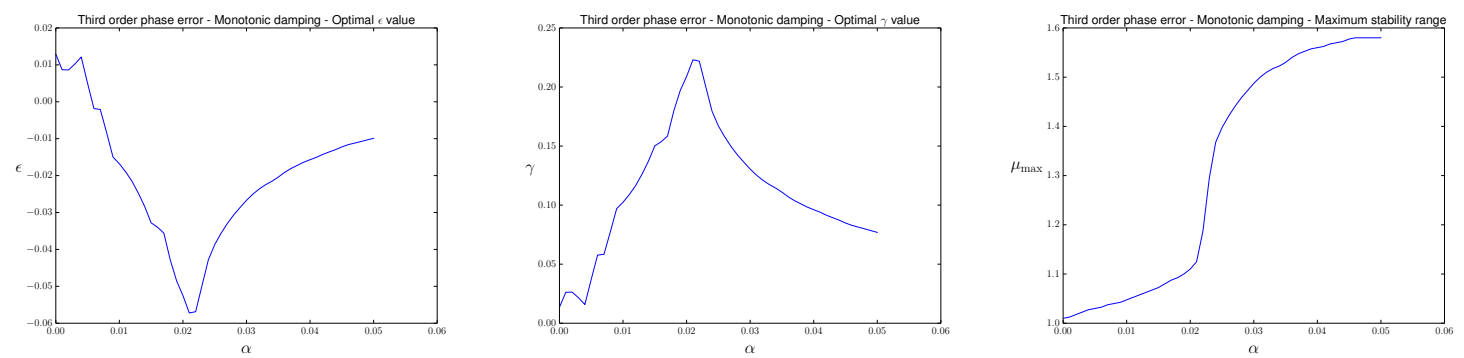

Figure B.13: Time stepping with third order phase error: optimal $\epsilon$ value for maximum stability range and corresponding $\gamma$ (given by (B.3)) and stability range ( $\mu_{\max }$ value).

In absence of diffusion the time stepping algorithm has a relatively strong stability constraint $\left(\mu_{\max }(\alpha=\right.$ $0) \approx 1$ ). The range of stability increases with the diffusion coefficient.

In order to increase the stability limit, the original ROMS time step algorithm relaxes the third order accuracy by removing the constraint (B.3). Doing so and maintaining the same maximum phase error than the original scheme leads to a two parameters optimization problem $(\epsilon, \gamma)$. Figure (B.14) shows the corresponding results.
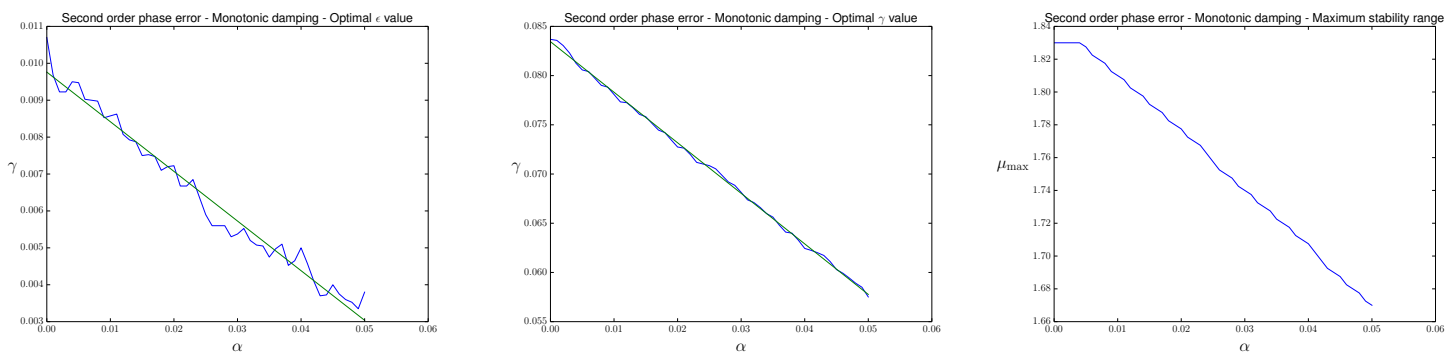

Figure B.14: Time stepping with second order phase error: optimal $\epsilon, \gamma$ values for maximum stability range and stability range ( $\mu_{\max }$ value).

Relaxing constraint (B.3) indeed allows a large increase of the stability range. The green lines on Fig. (B.14) 
represent polynomial fits to optimal values of $\epsilon, \gamma$ given by the following formulae:

$$
\epsilon=0.00976186-0.13451357 \alpha, \quad \gamma=0.083445-0.513584 \alpha
$$

Characteristic roots of the algorithms with third order phase error are shown on figure (B.15).
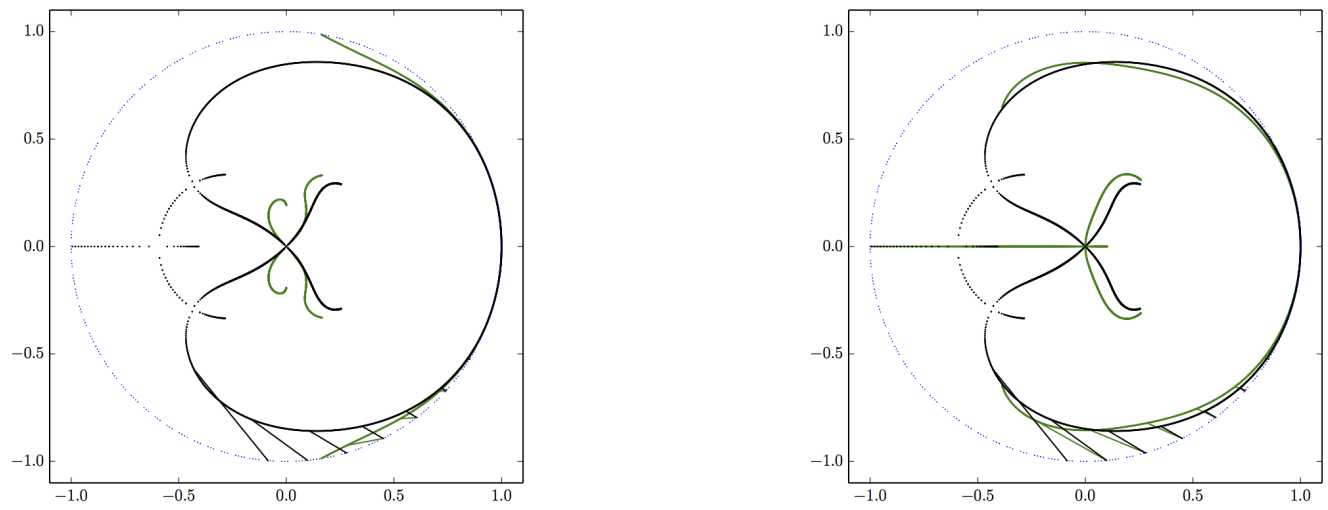

Figure B.15: Characteristic roots (in green) for third order phase error for optimized $\epsilon$ and for $\alpha=0$ (i.e. without second order damping, left, $\mu_{\max }=1.01$ ) and $\alpha=0.045$ (right, $\mu_{\max }=1.58$ ). The black curves correspond to the roots of the original ROMS algorithm $\left(\mu_{\max }=1.78\right)$.

Without second order diffusion (Fig. (B.15), left), it is not possible to strictly enforce monotonic damping. The resulting scheme is third order accurate and closed to an AB3-AM3 scheme. Note that even at low frequencies, the ROMS time step has only a second order phase error; and at medium frequencies the phase error of the ROMS scheme is much smaller that the formally third order accurate scheme.

Relaxing the constraint of third order phase error at low frequencies, characteristic roots of the algorithms with second order phase error are shown on figure (B.16).
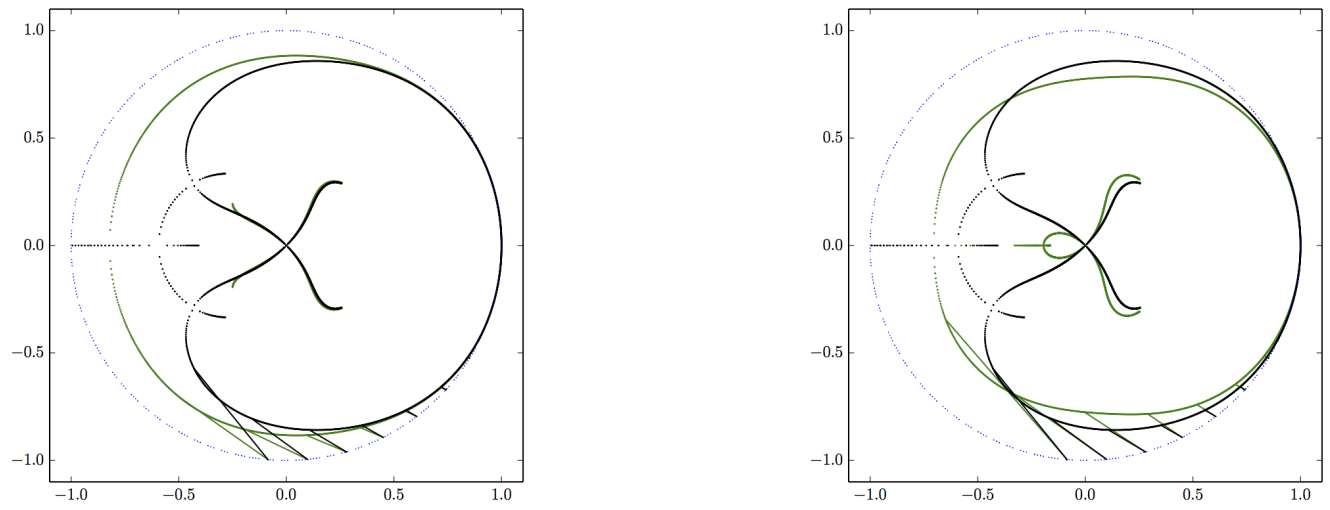

Figure B.16: Characteristic roots (in green) for second order phase error for optimized $\epsilon, \gamma$ and for $\alpha=0$ (i.e. without second order damping, left, $\mu_{\max }=1.83$ ) and $\alpha=0.045$ (right, $\mu_{\max }=1.69$ ). The black curves correspond to the roots of the original ROMS algorithm $\left(\mu_{\max }=1.78\right)$.

When $\alpha=0$ (no second order damping), the optimal (unapproximated by (B.4)) coefficients given by are 
$\epsilon=0.0107, \gamma=0.0836842$, the resulting algorithm is closed to the ROMS original algorithm $(\epsilon=0.013, \gamma=$ 0.0880). The resulting algorithm has a slightly enlarged stability range and is less dissipative at the price of a slightly higher phase error.

375 With $\alpha=0.045$, the optimized algorithm (Fig. (B.16), right, green) is, as expected, more dissipative than the original algorithm while the phase errors are almost identical.

In conclusion, the optimized scheme with parameters $\beta=0.281105, \epsilon, \gamma$ given by (B.4) and $\delta$ given by (B.2) is formally first order in time with (as required) second order damping $\left(|\lambda|=1-\alpha \mu^{2}+\mathcal{O}\left(\mu^{4}\right)\right)$ with a range of stability and a phase error closed to the original ROMS scheme. 